\title{
Stability Analysis of Position Datum for Real-Time GPS/BDS/INS Positioning in a Platform System with Multiple Moving Devices
}

\author{
Weiming Tang ${ }^{1,2}{ }^{\mathbb{D}}$, Yangyang $\mathrm{Li}^{1}$, Chenlong Deng ${ }^{1, *} \mathbb{C}$, Xuan Zou ${ }^{1}$, Yawei Wang ${ }^{1}$ and Kepei Qi ${ }^{1}$ \\ 1 GNSS Research Center, Wuhan University, 129 Luoyu Road, Wuhan 430079, China; \\ wmtang@whu.edu.cn (W.T.); yyangli@whu.edu.cn (Y.L.); zxuan@whu.edu.cn (X.Z.); \\ grcwongyw2016@whu.edu.cn (Y.W.); kepqi@whu.edu.cn (K.Q.) \\ 2 Collaborative Innovation Center of Geospatial Technology, 129 Luoyu Road, Wuhan 430079, China \\ * Correspondence: c.deng@whu.edu.cn
}

Citation: Tang, W.; Li, Y.; Deng, C.; Zou, X.; Wang, Y.; Qi, K. Stability Analysis of Position Datum for Real-Time GPS/BDS/INS Positioning in a Platform System with Multiple Moving Devices. Remote Sens. 2021, 13, 4764. https://doi.org/10.3390/ rs13234764

Academic Editor: Yunbin Yuan

Received: 19 September 2021

Accepted: 23 November 2021

Published: 24 November 2021

Publisher's Note: MDPI stays neutral with regard to jurisdictional claims in published maps and institutional affiliations.

Copyright: (C) 2021 by the authors. Licensee MDPI, Basel, Switzerland. This article is an open access article distributed under the terms and conditions of the Creative Commons Attribution (CC BY) license (https:// creativecommons.org/licenses/by/ $4.0 /)$.

\begin{abstract}
The rapid development of unmanned aerial vehicles (UAVs) in recent years has promoted their application in various fields, such as precise agriculture, formation flight, etc. In these applications, the accurate and reliable real-time position and attitude determination between each moving device in the same platform system are the key issue for safe and effective cooperative works. In traditional ways, static reference stations should be set up near the platform to keep the stable position datum of the platform system. In this paper, we abandoned the static stations and expected to achieve stable position datums with the platform system itself. To achieve this goal, we proposed an improved method based on both the Global Positioning System (GPS)/Beidou Navigation Satellite System (BDS) data and the inertial navigation system (INS) data to obtain precise positions of the moving devices. The time-differenced carrier phase (TDCP) was used to get the position variations and update the positions over time, and then, the INS data was integrated to further improve the accuracy and reliability of the updated positions; thus, this method is denoted as the TDCP/INS method. To evaluate the performance of this method and compare it with the traditional single-point positioning (SPP) method and the Kalman filtered SPP (KFSPP) method, a field vehicle experiment was conducted, and the position results achieved from these three methods were compared with those from the tightly combined real-time kinematic positioning (RTK)/INS method, where centimeter-level accuracy was obtained and regarded as the reference. The quantitative analysis where the position variations were evaluated and the qualitative analysis where the vehicle trajectories in three typical urban driving scenarios were discussed were both made for the three methods. The numerical results showed that the accuracy of the position variations from the SPP, KSPP, and TDCP methods was at the meter level, while that from the TDCP/INS method improved to the centimeter level, and the accuracies were $1.9 \mathrm{~cm}, 2.9 \mathrm{~cm}$, and $3.1 \mathrm{~cm}$ in the east, north, and upward directions. The trajectory results also demonstrated a perfect consistency of the driving positions between the TDCP/INS method and the reference. As a contrast, the trajectories from the SPP and KFSPP methods had frequent jumps or sways when the vehicle drove along a large, curved road, turned at a crossroad, and passed under an urban viaduct.
\end{abstract}

Keywords: position datum; GPS/BDS; INS; time-differenced carrier phase (TDCP); position variation

\section{Introduction}

With the rapid development of fifth-generation (5G) communication, autonomous driving, the Internet of Things (IoT) and other information technologies, the era of the Internet of Everything is coming. The demands for real-time precise and reliable relative positioning services in multiple moving equipment are increasing, especially in application areas requiring short distances and high speeds, such as the formation of aircrafts, vehicles, and ships. This brings a higher challenge to real-time high-precision positioning 
services. Multiple moving equipment usually form a joint platform system, and their data are exchanged to realize self-organized movement, i.e., collaborative work like bees. The accurate and reliable real-time position and attitude information of each device are the key for the safe and effective running of the moving platform system. Besides, to ensure a safe distance and cooperative operation between two devices, only the synchronous precise relative position and attitude information are sufficient. Although precise point positioning (PPP) technology can obtain centimeter-level absolute positioning results with only one receiver, the long initialization time and the dependency on additional network communications and augmentation products in real-time processing limit its application [1-3]. However, real-time kinematic (RTK) positioning technology, which utilizes differential information from a reference station, can realize fast and easily implemented real-time centimeter-level relative positioning; thus, it is widely employed in numerous real-time high-precision applications [4].

According to the move status of the reference station, the RTK technique can be divided into two modes, i.e., the "static-to-kinematic" mode, where a static reference station with known coordinates is required to transmit the differential data to moving devices, and the "kinematic-to-kinematic" mode, where both stations at the baseline are moving for real-time relative positioning. In the majority of contributions on kinematic positioning for a joint moving platform system, the "static-to-kinematic" mode is applied, and each moving device acquires centimeter-level positioning results relative to the nearby static reference station once the integer ambiguity is correctly fixed. Through the coordinate transfer, the precise relative position between any two moving devices in this platform system can be determined. However, this strategy relies heavily on the static reference station. The unexpected interruption of the communication link or data processing in the static reference station will paralyze the entire moving platform system. In the meantime, since the reference station is static, the coverage area of the sent differential data is limited, and the performance of the ambiguity initialization and positioning accuracy will decrease if the moving platform system is away from the static reference station. Furthermore, the real-time relative positioning of each device in the moving platform system requires large data transmission and communication ability in the static reference station if there are lots of moving devices, which means a higher cost for equipment at the static reference station. Alternatively, the other "kinematic-to-kinematic" mode has been gradually attracting more and more attention [5].

In the "kinematic-to-kinematic" mode, any moving device in the platform system can be selected as the reference station for real-time positioning relative to the other moving devices. In open-source software RTKLIB version 2.4.2, Takasu and Yasuda realized the kinematic-to-kinematic relative positioning in the module's "moving baseline" [6]. Singlepoint positioning (SPP) was used first to obtain the approximate coordinates of the reference moving station; then, Kalman filtering was applied to estimate the position, velocity, and single-differenced ambiguity, and finally, the baseline solution was achieved once the ambiguity was fixed [7]. However, the actual performance of the positioning was not inspiring due to the unsatisfactory success rate of the ambiguity resolution.

It should be noted that, although this software is very popular and lots of the literature on kinematic data processing with RTKLIB can be found, unfortunately, most of them only utilized the "PPP kinematic" or "static-to-kinematic" modes, and works in the "kinematic-to-kinematic" mode can hardly be found. For example, Berber et al. analyzed the results from other open-source online services together with RTKLIB in the PPP mode [8], Gurturk et al. analyzed the results of flight data from RTKLIB in both the PPP with ambiguity resolution and postprocessing kinematic methods [9], and $\mathrm{Wu}$ et al. analyzed the positioning performance of smartphone data based on RTKLIB with, also, the PPP algorithm [10]. Hemant et al. utilized RTKLIB, along with two stations for kinematic positioning, but the base station was static; thus, the "static-to-kinematic" mode was applied [11]. 
Besides the results from RTKLIB, Luo constructed the optimal Delaunay triangulation in the platform system and selected the optimal baseline as the constraint condition to accelerate the ambiguity convergence [12]. Based on this approach, MultiKIN software was developed for relative positioning in a joint moving platform system. Dong et al. (2020) considered a data delay during the movement of a reference station and presented an asynchronous RTK (ARTK)/time-differenced carrier phase (TDCP) and inertial navigation system (INS)-integrated method, and a decimeter-to-centimeter-level higher-rate $(\geq 100 \mathrm{~Hz})$ relative positioning was obtained [13].

It was obvious that all the estimated coordinates of the moving devices during the whole observation were compatible and consistent in the "static and kinematic" mode, since all their coordinates referred to the only static reference station with a fixed coordinate, which means the position datum of the entire moving platform system was fixed to the static reference station and would not change over time. In this way, all devices in the entire moving platform system could achieve their consecutive trajectory by epochs [14,15]. However, in the "kinematic-to-kinematic" mode, a simple epoch-independent SPP cannot reflect the precise position change of the moving reference station due to the accuracy limitation of the SPP. Under these circumstances, although centimeter-level relative positioning can be reached between the moving reference station and the moving rover station in each epoch, their absolute coordinates over time will have frequent jumps along the true moving direction, which is caused by the positioning errors of the SPP. Even if Kalman filtering is applied to smooth the coordinates, the accuracy of the positioning results achieved by the pseudo-range-only positioning is still unsatisfactory [16]. In other words, the position datum in the moving platform system is very unstable during the entire movement, which leads to an inconsistency between the positioning results and the actual motion trajectory of each moving device in the platform system. Although this problem can be solved by the precise real-time PPP, the longtime ambiguity initialization and the requirements of the additional GPS/BDS state space representation (SSR) corrections described above make it unpractical to apply this technique in "kinematic-to-kinematic" positioning $[17,18]$.

Considering that the position variations of the moving reference station can be accurately determined if observables with high sampling rates $(\geq 1 \mathrm{~Hz})$ are processed, the stability of the position datum, which refers to the accuracy of the position variations of the moving reference station, can be maintained. Usually, three different methods can obtain the position variations, including position difference (PD), Doppler measurements, and TDCP [19]. The PD method differences two consecutive position solutions and is very easy to implement, but the accuracy of the determined position variations relies much on the position accuracy, and the change of the satellite number will introduce a discontinuity of the position. The Doppler measurements produced by the receiver satellite relative motion enable an average velocity accuracy of around $2 \mathrm{~cm} / \mathrm{s}$, and the position variations can only be calculated by multiplying the epoch interval. However, the velocity of the moving device may vary during the epoch interval, and the product cannot accurately reflect the actual position variation. Therefore, when the moving devices accelerate rapidly, the accuracy cannot be guaranteed [20]. On the contrary, with the TDCP method, differences in the carrier phase observables between consecutive epochs can directly lead to position variations of the moving devices [21]. Meanwhile, for data with a sampling rate no lower than $1 \mathrm{~Hz}$, the TDCP method almost removes the time-correlated errors, such as the clock and ephemeris errors in the satellite segment and the atmospheric delays, as well as eliminates the integer ambiguity [22]. In this way, centimeter-level position variations can be achieved [23]. As a result, the precise positions variation obtained by the TDCP method in kinematic relative positioning can be used to transfer the coordinates of the moving reference station along with the time and, thus, ensure the stability of the position datum.

On the other hand, we should realize that, in environments with severe signal obstructions such as urban canyon areas, the accuracy, reliability, and continuity of Global Navigation Satellite System (GNSS)-only real-time relative positioning cannot be guaranteed. Usually, the GNSS system is integrated with the inertial navigation system (INS) to 
improve the navigation and positioning performance [24,25]. Besides, considering the cost of the traditional inertial measurement unit (IMU) equipment, the low-cost micro-electromechanical system (MEMS) becomes an ideal alternative in the civil consumer market due to its small size, high rate, and good dynamic performance, and it is more and more popular in precise kinematic positioning applications such as unmanned aerial vehicles (UAVs), automatic driving, etc. [26-28].

In view of the current instability of position datum in "kinematic-to-kinematic" relative positioning in a platform system with multiple moving devices, in this paper, we will propose an integrated method in which a loose combination (LC) between the GPS/BDS TDCP method and the INS is employed to maintain the stability of the position datum in the moving platform system. The consistency between the positioning results and the actual motion trajectory further ensures the safety distance and collaborative operation between the moving devices. The performance of the proposed method will be analyzed and compared with the tight combined (TC) RTK/INS results [29,30].

\section{Materials and Methods}

\section{1. $S P P$}

In a simple GPS or BDS SPP model, the code observation equation of any satellite is expressed as:

$$
P=\rho+c \cdot\left(d t_{r}-d t^{s}\right)+\delta_{r}-\delta^{s}+\text { Orb }+ \text { Ion }+ \text { Trop }+\varepsilon_{P},
$$

where $P$ refers to the code observation. $\rho$ is the geometric distance between the satellite and the receiver. $c$ is the speed of light. $d t$ and $\delta$ are the clock error and the code hardware delay, whose subscript $r$ and superscript $s$ are the receiver and the satellite, respectively. Orb denotes the orbital error, and Ion and Trop are the ionospheric delay and the tropospheric delay, respectively. $\varepsilon_{P}$ indicates the multipath effect and the measurement noise.

For at least four tracked satellites, the linearized observation equation in the matrix could be written as follows:

$$
P=A X+L,
$$

where $P$ represents the observation vector of the code, $A$ is the coefficient matrix, and $A=(B, E)$, where $B$ is the direction matrix from the receiver to the satellites, and $E$ is a column vector with all elements being 1 . The parameters to be estimated $X$ include the coordinate corrections and the clock error of the receiver, i.e., $\boldsymbol{X}=\left(d x, d y, d z, d t_{r}\right)$. Herein, the hardware delays of the receiver and the satellite are absorbed into the receiver clock and cannot be separated. The remaining matrix $L$ is the calculated ranges between the receiver and the satellites, which include the initial geometry distances between the receiver and the satellites, the modeled satellite clock errors, and corrections of the ionospheric delays and tropospheric delays by empirical models such as the Klobuchar model [31] and the Saastamoinen model [32], respectively.

Furthermore, in a combined GPS/BDS model, the intersystem code bias (ISCB) in different systems should be considered, and the estimators in Equation (2) are modified to $\left(d x, d y, d z, d t_{r}^{G}, d t_{r}^{C}\right)$, where the superscripts G and C denote the GPS system and BDS system, respectively. As a result, the least-squares (LS) estimation can only be employed for more than five tracked satellites, and the specific observation equation can be expressed as

$$
\left[\begin{array}{l}
P^{G} \\
P^{C}
\end{array}\right]=\left[\begin{array}{ccc}
B^{G} & E^{G} & 0 \\
B^{C} & 0 & E^{C}
\end{array}\right] X+L,
$$

As for the stochastic model, all observations are regarded as independent, and their variances are determined by a simple satellite elevation-related model, expressed as

$$
\sigma^{2}=\left\{\begin{array}{c}
\sigma_{0}^{2} / \sin (E) E \geq 30^{\circ} \\
\sigma_{0}^{2} / \sin ^{2}(E) E<30^{\circ}
\end{array}\right.
$$


where $\sigma_{0}$ is the a priori code noise of $1 \mathrm{~m}$ for both GPS and BDS, and $E$ is the satellite elevation. Besides, the weights of different systems are determined separately and formed as a diagonal matrix. Therefore, the weight matrix of the combined GPS/BDS system $\boldsymbol{P}_{\boldsymbol{W}}$ is expressed as

$$
P_{W}=\left[\begin{array}{cc}
P_{W}^{G} & 0 \\
0 & P_{W}^{C}
\end{array}\right]
$$

\subsection{Kalman-Filtered SPP (KFSPP)}

For a quick-moving device, the only position is not enough to fully describe its motion state, and the velocity is an important supplement. By acquiring the velocity information, one can establish an approximate kinematic equation for the moving device, and its state of motion can be precisely depicted. For this reason, we can estimate the velocity of the moving device by using Doppler measurements.

The user satellite relative motion leads to changes in the phase and frequency, or the Doppler shift. Scaling the Doppler shift by the carrier wavelength, the code changing rate observation can be obtained, whose linearized form can be written as follows:

$$
\lambda D=l \cdot\left(v_{x}-v^{x}\right)+m \cdot\left(v_{y}-v^{y}\right)+n \cdot\left(v_{z}-v^{y}\right)+c \cdot d \bar{t}_{r}
$$

where $\lambda$ is the wavelength of the carrier phase, $D$ is the Doppler shift value, $(l, m, n)$ is the coefficient matrix, $\left(v_{x}, v_{y}, v_{z}\right)$ and $\left(v^{x}, v^{y}, v^{z}\right)$ are the velocities of the receiver and the satellite, and $d \bar{t}_{r}$ is the receiver clock drift.

Since the motion state of the moving device can be described, the Kalman filtering (KF) technique can then be used to determine its position and velocity together with the observation equations of the code and code changing rate. Provided that the velocity of the moving device is constant between consecutive epochs, its motion state can then be expressed as

$$
\boldsymbol{X}_{k+1}=\boldsymbol{\Phi}_{k+1, k} \boldsymbol{X}_{k}+\boldsymbol{w}_{k},
$$

where $k$ represents the epoch. $\boldsymbol{X}$ is the vector of the device motion state, and $\boldsymbol{X}=$ $\left(x, y, z, d t_{r}^{G}, d t_{r}^{C}, v_{x}, v_{y}, v_{z}, d \bar{t}_{r}^{G}, d \bar{t}_{r}^{C}\right) . \boldsymbol{\Phi}$ is the transition matrix between the consecutive epochs. According to the kinematical equation for a moving object with a constant velocity, the transition matrix can be expressed as

$$
\boldsymbol{\Phi}_{k+1, k}=\left[\begin{array}{cc}
\boldsymbol{I} & \Delta t \cdot \boldsymbol{I} \\
0 & \boldsymbol{I}
\end{array}\right],
$$

where $I$ is a $5 \times 5$ identity matrix, and $\Delta t$ is the time interval. $w$ is the process noise vector whose covariance matrix is determined as follows:

$$
\boldsymbol{Q}=\left[\begin{array}{cccc}
\boldsymbol{S}_{v} \Delta t^{3} / 3 & \mathbf{0} & \boldsymbol{S}_{v} \Delta t^{2} / 2 & \mathbf{0} \\
\mathbf{0} & \boldsymbol{S}_{t} \Delta t+\boldsymbol{S}_{f} \Delta t^{3} / 3 & \mathbf{0} & \boldsymbol{S}_{f} \Delta t^{2} / 2 \\
\boldsymbol{S}_{v} \Delta t^{2} / 2 & \mathbf{0} & \boldsymbol{S}_{v} \Delta t & \mathbf{0} \\
\mathbf{0} & S_{f} \Delta t^{2} / 2 & \mathbf{0} & S_{f} \Delta t
\end{array}\right]
$$

where $S_{v}$ is a $3 \times 3$ diagonal matrix representing the power spectral density (PSD) of the acceleration, and $S_{t}$ and $S_{f}$ are $2 \times 2$ diagonal matrices denoting the PSD of the clock bias and clock drift. Herein, all the diagonal elements in $S_{v}, S_{t}$, and $S_{f}$ are set to an empirical value of 0.05 . Besides, the variance of the code and the Doppler observations are set to 4.5 and 0.18 , respectively.

Using at least five simultaneous code and Doppler measurements, the position, velocity, clock offset, and clock drift of the moving device could be obtained by the KF technique. Compared with the simple SPP technique, Doppler observations are involved, and Kalman filtering is employed in KFSPP; thus, better accuracy of the positioning is expected. 


\subsection{TDCP}

In the above KFSPP, the velocity is determined, and the kinematic equation is utilized to improve the accuracy of the position. However, the assumption that the velocity of the moving device is constant between consecutive epochs is probably unrealistic, and the velocity of the device may vary from one epoch to another. Alternatively, the TDCP observation can provide another way to directly determine the position variations between adjacent epochs.

The TDCP observation is derived from the original phase observation, which can also provide receiver satellite distance estimations, and the original phase observation equation in meters can be expressed as [33]:

$$
\lambda \varphi=\rho+c \cdot\left(d t_{r}-d t^{s}\right)+\zeta_{r}-\zeta^{s}+\text { Orb }- \text { Ion }+ \text { Trop }-\lambda N+\varepsilon_{\varphi},
$$

where $\lambda$ is the wavelength, $\varphi$ is the carrier phase in cycles, $\zeta$ is the phase hardware delay, $N$ is the integer ambiguity, and $\varepsilon_{\varphi}$ includes the multipath and the phase noise. The TDCP observation is then obtained by differencing the carrier phase observations of the same satellite at two successive epochs: $t$ and $t+1$, which can be expressed as:

$$
\lambda \cdot \Delta \varphi=\Delta \rho+c \cdot\left(\Delta d t_{r}-\Delta d t^{s}\right)+\Delta \zeta_{r}-\Delta \zeta^{s}+\Delta \text { Orb }-\Delta \operatorname{Ion}+\Delta \operatorname{Trop}+\varepsilon_{\Delta \varphi},
$$

where $\Delta$ represents the time-differencing operation. Assuming that no cycle slips occur between these two epochs, in the TDCP observation, the unknown integer ambiguity is totally canceled, because it is a constant. Most of the other common errors such as $\Delta d t^{s}, \Delta \zeta_{r}, \Delta \zeta^{s}, \Delta O r b, \Delta I o n$, and $\Delta T r o p$ vary slowly with the time and can also be almost eliminated in a very short time interval [34]. Therefore, the TDCP observation equation can be simplified to

$$
\lambda \cdot \Delta \varphi=\Delta \rho+c \cdot \Delta d t_{r}+\varepsilon_{\Delta \varphi}
$$

Just like Equation (2), the linearized TDCP observation equation in the matrix can be obtained if at least 5 satellites are continuously tracked at two consecutive epochs for the combined GPS/BDS system. Then, the LS estimation can be applied to obtain the position variations $(\Delta x, \Delta y, \Delta z)$ and the clock drift of the moving device. The position of the moving device at the current epoch can be easily derived from the position at the previous epoch as

$$
\left[\begin{array}{l}
x_{k+1} \\
y_{k+1} \\
z_{k+1}
\end{array}\right]=\left[\begin{array}{l}
x_{k} \\
y_{k} \\
z_{k}
\end{array}\right]+\left[\begin{array}{l}
\Delta x \\
\Delta y \\
\Delta z
\end{array}\right]
$$

Since carrier-phase observations with millimeter-level noise are used, there is a promising probability of centimeter-level position variations being obtained. In the meantime, the carrier phase also brings possible cycle slips. To handle this problem, the TurboEdit method [35] was first utilized for data preprocessing. Then, the posterior mean error of the unit weight was checked after a standard least-square estimation. If this value is remarkably increased at one epoch, it means the cycle slip occurs in this epoch, and a robust least-square estimation was thereby applied to detect the specific satellites with cycle slips. At last, additional parameters were added in the observation equation to resolve these cycle slips. In this way, the possible cycle slip is detected and repaired.

\subsection{TDCP/INS}

Despite the high precision of carrier-phase observations in the TDCP method, the signal is susceptible to the environment around the station. In order to ensure a high reliability and availability of the moving reference position, in this study, the INS data will be collected and integrated with the GPS/BDS data, and an improved integrated method will be proposed. Since this method combines the results from the TDCP method and the INS data, we call this method the TDCP/INS method. 
In the TDCP/INS method, the precise position variations are determined via the TDCP method, and the position at the current epoch is updated through Equation (13). Once the updated position is achieved, a loose combination integration (LC) between the position information and the velocity/acceleration information from the INS data can be employed. In this way, the stability and reliability of the position results in complex urban environments can be further improved.

In the INS error model, only the stochastic biases of the gyros and accelerometers are to be considered and are modeled as the first-order Gauss-Markov process. As a result, a 15-state INS dynamic model, including the three-dimensional attitude $\psi$, velocity $\delta v$, position $\delta \boldsymbol{r}$, gyros bias $\delta \boldsymbol{b}_{\boldsymbol{g}}$, and acceleration bias $\delta \boldsymbol{b}_{\boldsymbol{a}}$, is established. The form of its state equation is the same as that in Equation (7), but the parameter to be estimated is modified to $\boldsymbol{X}_{k}=\left(\boldsymbol{\psi}, \delta \boldsymbol{v}, \delta \boldsymbol{r}, \delta \boldsymbol{b}_{g}, \delta \boldsymbol{b}_{\boldsymbol{a}}\right)$. The definitions of the transition matrix $\boldsymbol{\Phi}$ and the process noise vector $\boldsymbol{w}$ are referred to in Reference [23], where the variances of the position in three different directions were all set as $9 \mathrm{~cm}^{2}$, considering the centimeter-level accuracy of the position variations.

The observation equation of the TDCP/INS model is constructed based on the updated position results determined by the combined GPS/BDS system and the corresponding information predicted by INS data and can be expressed as:

$$
\mathbf{Z}_{k}=\boldsymbol{H}_{k} \boldsymbol{X}_{k}+\varepsilon_{k}
$$

where $Z_{k}$ is the observation vector, expressed as

$$
\boldsymbol{Z}_{k}=\left[\begin{array}{l}
\boldsymbol{r}_{G N S S}-\boldsymbol{r}_{I N S} \\
\boldsymbol{v}_{G N S S}-\boldsymbol{v}_{I N S}
\end{array}\right],
$$

where $r$ and $v$ represent the position and velocity vectors obtained by GPS/BDS and INS, respectively. $\boldsymbol{H}_{k}$ is the coefficient matrix, and $\varepsilon_{k}$ is the observation noise vectors. Based on the established state equation and the observation equation, the KF technique is then applied in integrated GPS/BDS/INS positioning to obtain the attitude, velocity, and position information.

Compared with the TDCP alone method, the INS data further ensures the reliability of the position results in complex urban environments. In this way, the stability of the position datum can be maintained.

\subsection{Method Comparison}

To demonstrate and compare the effects of the positioning results from different methods, we drew a sketch map showing the position information of one moving device along with the time, as seen in Figure 1. The positions obtained from different methods and the corresponding trajectories are displayed with dots and lines in different colors.

Specifically, the moving reference position at the beginning epoch $t$ can only be obtained by the SPP method; thus, there is inevitably an obvious deviation between the calculated position and the true position caused by the positioning error of the SPP. This situation occurs for all methods, including the SPP, KFSPP, TDCP, and TDCP/INS.

In the subsequent epochs, the position variations are obtained by the TDCP alone or the TDCP/INS method, and the moving position at the next epoch is updated from Equation (13) and further improved through the integration with the INS data. Since the determined position variations are accurate and the improved position is reliable, the final positions will continuously keep almost the same bias from the true ones. Therefore, the trajectory from the TDCP alone or TDCP/INS method is approximately a translation of the actual trajectory, as is shown with the green dots and line in Figure 1. 


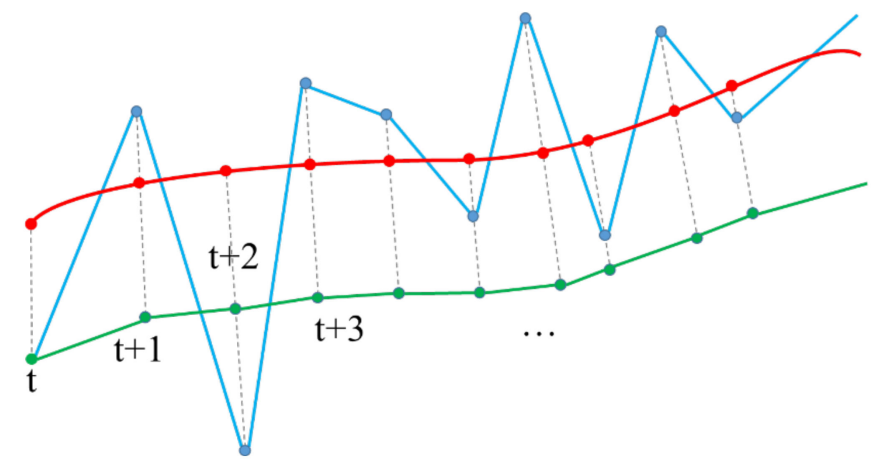

Figure 1. The illustration of the moving trajectories obtained via different approaches. The red dots and line represent the actual positions and trajectory, the blue dots and line denote the positions and trajectory obtained from the SPP or KFSPP, and the green dots and line are the positions and trajectory from TDCP alone or TDCP/INS.

On the contrary, the positions obtained by the SPP method have random errors of 3-10 $\mathrm{m}$. Even if the KF technique is applied in the KFSPP method, the random errors are hardly reduced to below $1 \mathrm{~m}$. Therefore, the position results obtained by these two methods show frequently jumps around the true positions at all the epochs, as we can see with the blue dots and line in Figure 1.

Although the trajectory by the TDCP alone or TDCP/INS method has a systematic bias from the actual trajectory, the bias is very stable along the timeline. For a platform system with multiple moving devices, the constant systematic bias does not matter, and the stability of the position datum can be maintained during the whole motion period.

\section{Results}

In this section, a vehicle field experiment is introduced when the GPS/BDS and INS data were collected. The satellite availability of the GPS and BDS and the position dilution of precision (PDOP) of the moving device during the observation are analyzed to reflect the observation environment. Then, a quantitative analysis is made, and the numerical results of the position variations acquired from the SPP, KFSPP, TDCP alone, and TDCP/INS are evaluated. Finally, a qualitative analysis is made to visually assess and compare the consistency of the motion trajectory obtained from different methods in three typical urban driving scenarios, which include going along a large, curved road, turning at a crossroad, and passing under an urban viaduct.

\subsection{Data Collection}

In order to analyze and assess the performance of the TDCP/INS method, nearly one-hour's vehicle field experiment was carried out on November 30th, 2020 in an urban environment of Wuhan, China. A K708 GNSS original equipment manufacturer (OEM) board manufactured by ComNav Technology Ltd. in China was used to collect the GPS/BDS data, and an ADIS16470 MEMS module manufactured by Analog Devices Inc. was used to synchronously collect the IMU data. The specifications of these two products and their configurations in use are listed in Tables 1 and 2, respectively. Since only the low-cost MEMS is applied, the noise of its observations is relatively larger than other expensive INS modules. On the other hand, no blunder detection or noise elimination was employed in this experiment, which may have limited the accuracy of the final results.

It should be noted that the sampling rate of the GNSS receiver was set to $1 \mathrm{~Hz}$, while that of the MEMS module was set to $100 \mathrm{~Hz}$, and only the results at synchronous epochs will be compared for the three methods. More details about the models and strategies applied in the data processing of the TDCP/INS method are shown in Table 3. 
Table 1. Specifications and configurations of the K708 GNSS OEM board.

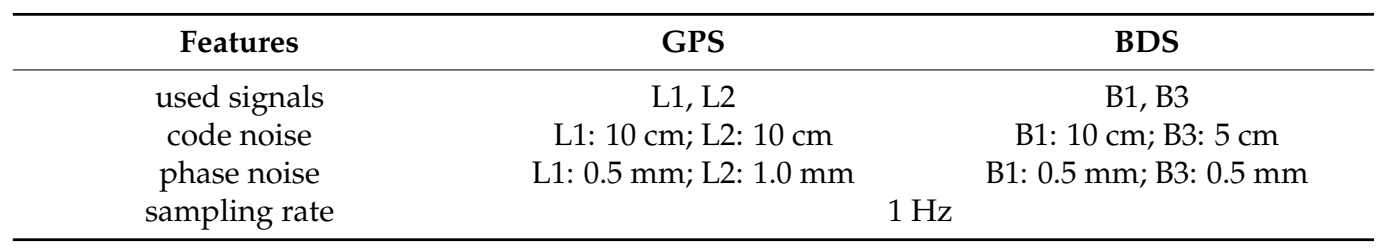

Table 2. Specifications and configurations of the ADIS16470 MEMS module.

\begin{tabular}{|c|c|c|}
\hline Features & gyroscope & accelerometer \\
\hline dynamic range & $\pm 2000^{\circ} / \mathrm{s}$ & $\pm 40 \mathrm{~g}$ \\
\hline in run stability & $8^{\circ} / \mathrm{h}$ & $13 \mu \mathrm{g}$ \\
\hline random walk & $0.34^{\circ} / \sqrt{h}$ & $0.037 \mathrm{~m} / \mathrm{s} / \sqrt{\mathrm{h}}$ \\
\hline output noise & $0.17^{\circ} / \mathrm{s}$ & $2.3 \mathrm{mg}$ \\
\hline sampling rate & & \\
\hline
\end{tabular}

Table 3. Models and strategies applied in data processing of the TDCP/INS.

\begin{tabular}{ccc}
\hline Parameter & Model & Constraint \\
\hline observation & GPS L1; BDS B1 & 0.02 cycles \\
observation weight & elevation weighted & $/$ \\
cutoff elevation & 15 degrees & $/$ \\
phase center pattern & igs14.atx & $/$ \\
ionospheric delay & Klobuchar model & $/$ \\
tropospheric delay & Saastamoinen model & broadcast \\
ephemerids & estimation + white noise & priori $10 \mathrm{~m}$ \\
receiver clock drift & fixed to IERS & $/$ \\
EOP &
\end{tabular}

The GNSS OEM board was connected with an antenna via the cables, and then, it was integrated with the MEMS module. Two sets of the integrated devices were placed on the roof rack of a sport utility vehicle. One was used in this study, and the other two were used for another purpose that was outside the scope of this study. The experimental vehicle and the installed equipment are displayed in Figure 2.

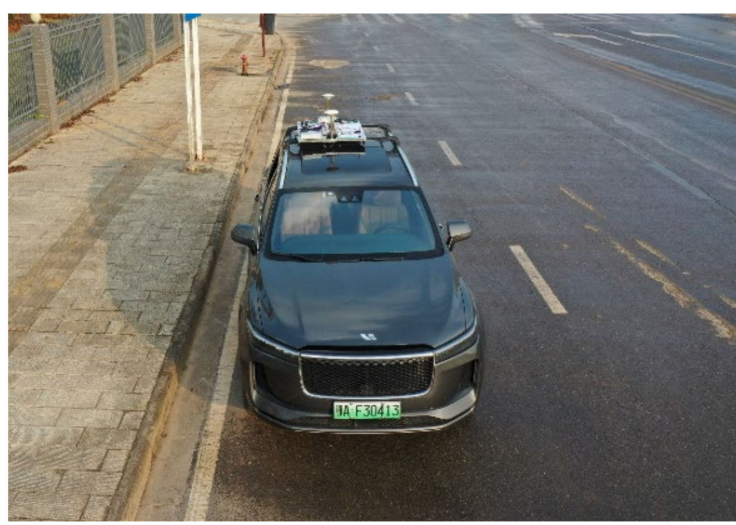

(a)

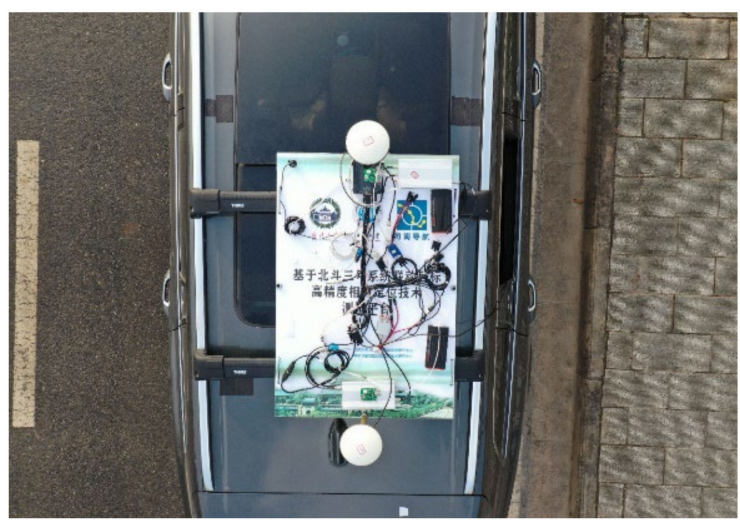

(b)

Figure 2. Experiment equipment, including: (a) experimental vehicle and (b) installed equipment on the roof rack.

To obtain accurate reference positions of the moving vehicle, one static reference station was set up in the Science Park of Wuhan University. The high-precision vehicle positioning and attitude determination system M39 developed by Wuhan MAP Space Time Navigation Technology Co., LTD was used, and the centimeter-level TC RTK/INS results were regarded as the "true" positions and velocities [36]. It should be noted that the 
proposed loosely combined TDCP/INS method belongs to the position domain and can directly obtain absolute positions without the information of the base station, while the tightly combined RTK/INS method belongs to the observation domain, and the achieved relative positions can only be changed to the absolute ones by the known coordinates of the base station.

The actual complete driving trajectory of the vehicle experiment from the TC RTK/INS method is shown in Figure 3, and those from the SPP, KFSPP, TDCP, and TDCP/INS methods are shown in Figure 4. From the actual trajectory, we can see that the vehicle turned at several corners at the beginning and then went straight along a long road. Additionally, the trajectories from the four other methods were similar to the actual one; this was due to the limited plotting scale on the maps.

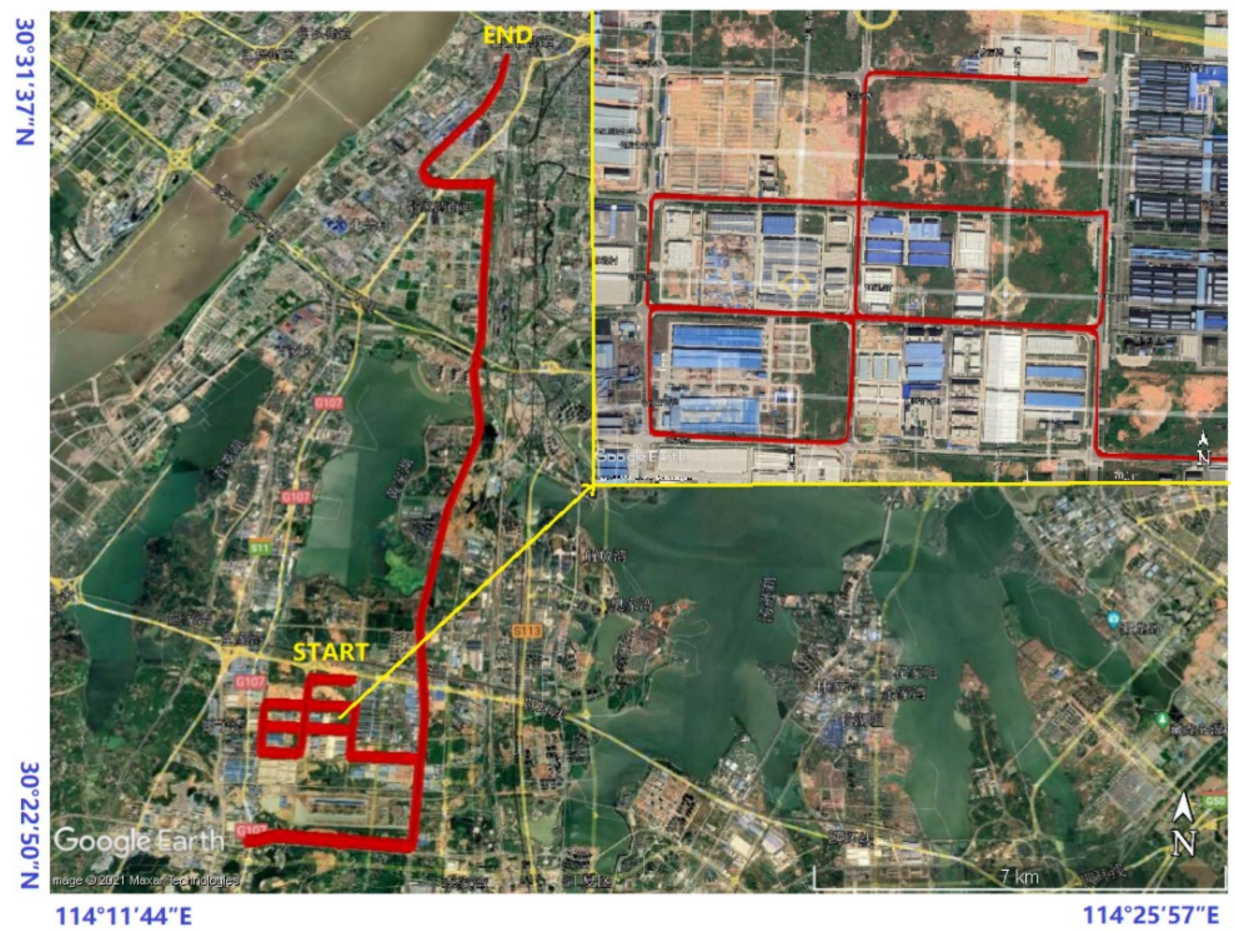

Figure 3. Actual complete vehicle trajectory from the TC RTK/INS method, as is shown by the red line. The longitude and latitude of the figure range from $114^{\circ} 11^{\prime} 44^{\prime \prime} \mathrm{E}, 30^{\circ} 22^{\prime} 50^{\prime \prime} \mathrm{N}$ to $114^{\circ} 25^{\prime} 57^{\prime \prime} \mathrm{E}$, $30^{\circ} 31^{\prime} 37^{\prime \prime} \mathrm{N}$.

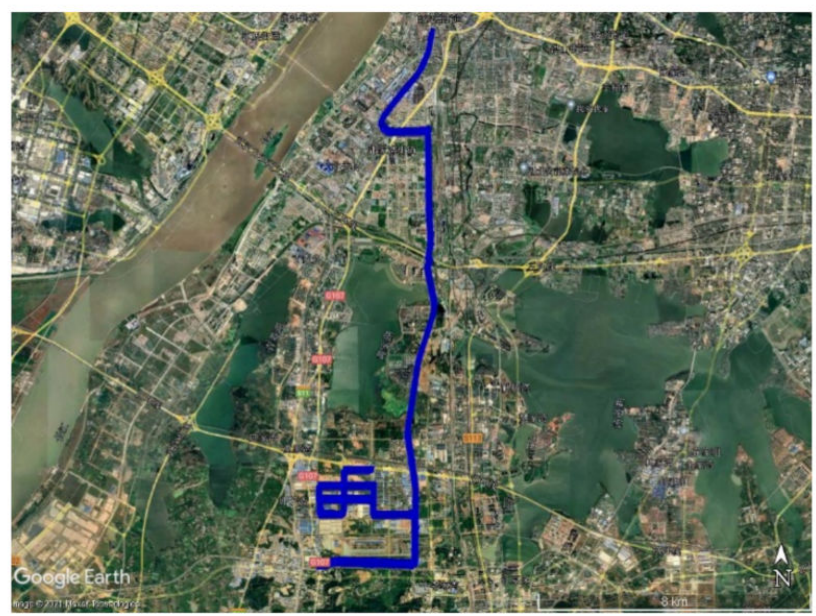

(a)

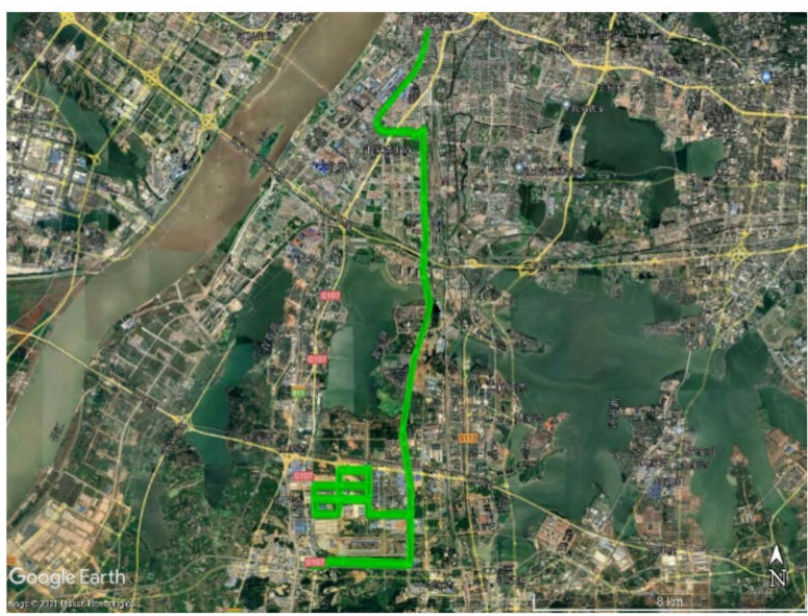

(b)

Figure 4. Cont. 


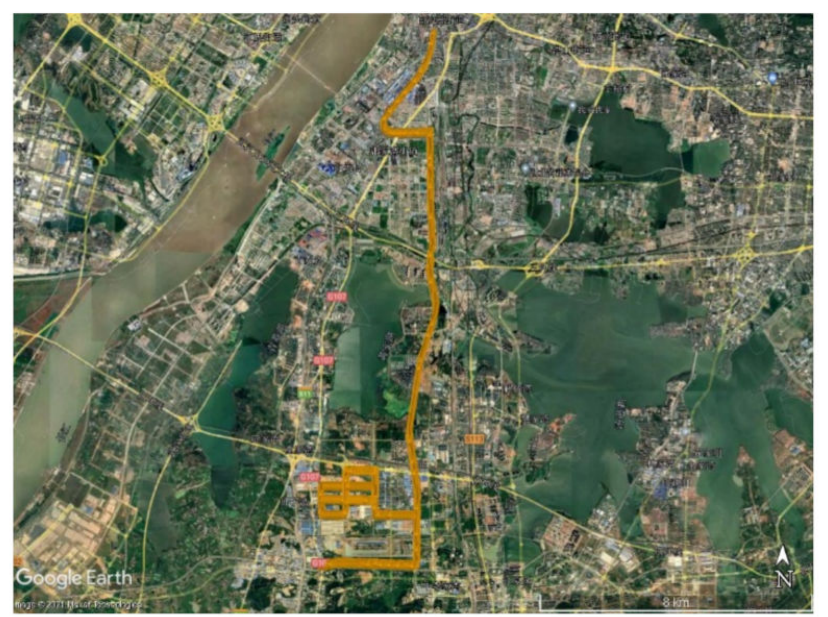

(c)

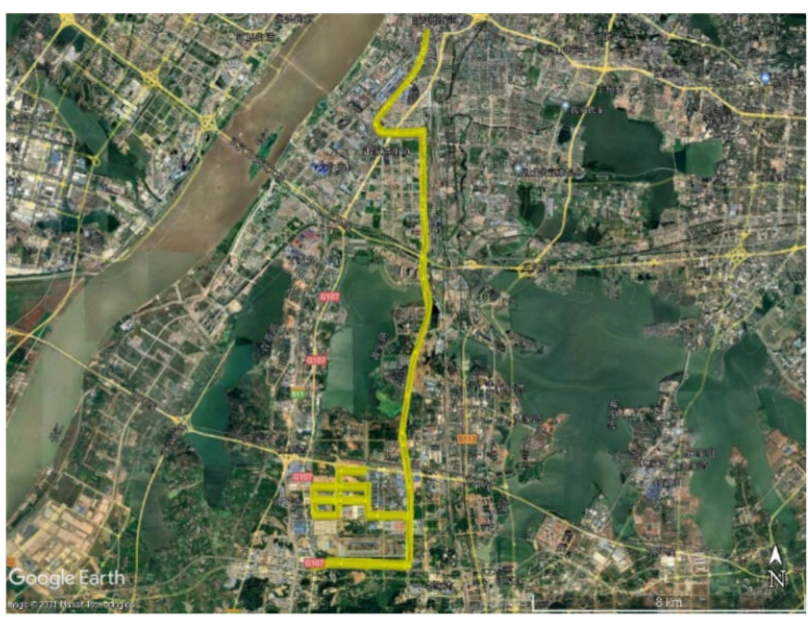

(d)

Figure 4. Complete vehicle trajectories from different methods: (a) the SPP results (blue line), (b) KFSPP results (green line), (c) TDCP results (orange line), and (d) TDCP/INS results (yellow line). The longitude and latitude range from $114^{\circ} 09^{\prime} 56^{\prime \prime} \mathrm{E}$, $30^{\circ} 22^{\prime} 05^{\prime \prime} \mathrm{N}$ to $114^{\circ} 24^{\prime} 39^{\prime \prime} \mathrm{E}, 30^{\circ} 31^{\prime} 28^{\prime \prime} \mathrm{N}$.

Meanwhile, the vehicle velocities from TC RTK/INS in the east (VE), north (VN), and upward (VU) directions along the timeline are shown in Figure 5. The velocity results also showed various scenarios of motion states during the nearly one-hour field test, which included frequent accelerations and decelerations.

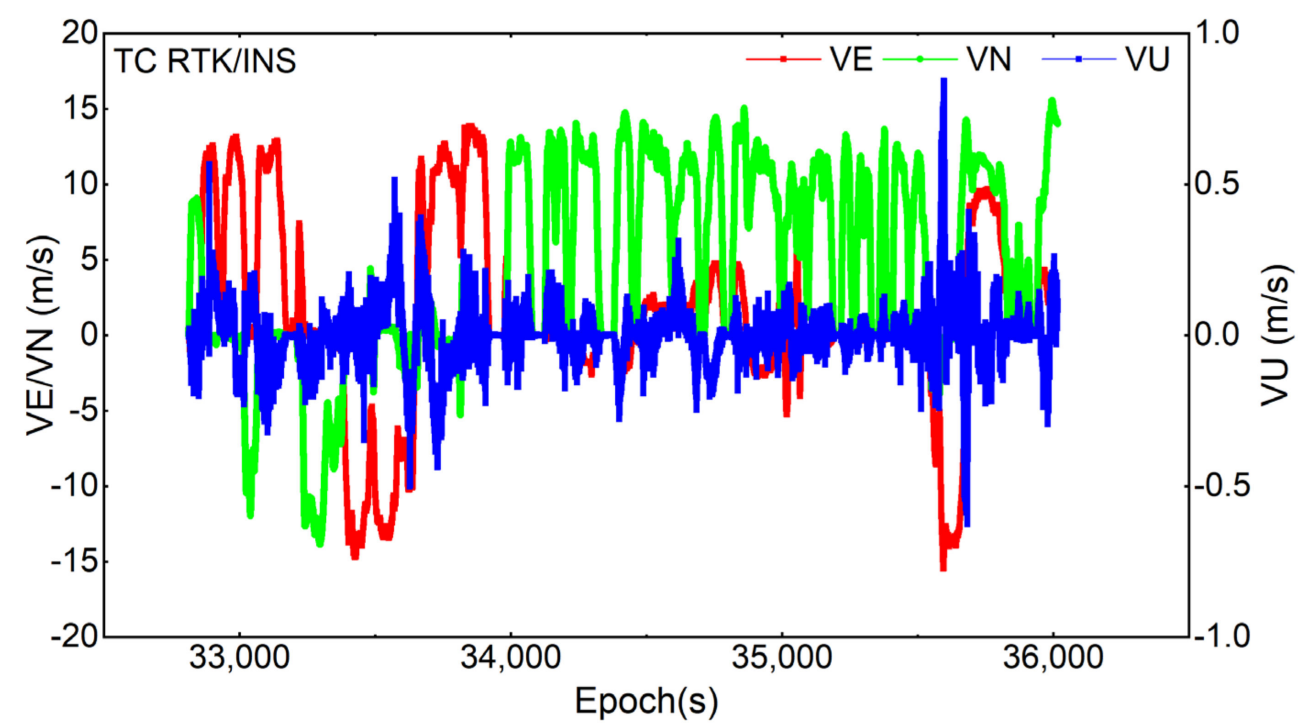

Figure 5. Velocity results in the east, north, and upward directions (VE/VN/VU) of the vehicle in the experiment by the TC RTK/INS.

\subsection{Satellite Availability and PDOP}

The satellite availability and the PDOP value are two important indicators when we evaluate the service performance of the system, and they have a significant impact on the final positioning accuracy. If more satellites are tracked and smaller PDOP values are achieved, the positioning results will be more accurate.

The tracked GPS, BDS-2, and BDS-3 satellites during the nearly one-hour field test are shown in Figure 6 when the cut-off elevation angle was set to $15^{\circ}$. Generally speaking, the observation conditions of the test were pretty good, because approximately 8 GPS satellites, 11 BDS-2 satellites, and 10 BDS-3 satellites could be tracked. On the other hand, we also found frequent signal interruptions for several satellites. This may be due to the changes or the interferences of the environment around the vehicle, such as the tall trees beside the 
road or the viaduct on the road. In these situations, the number of tracked satellites rapidly decreases, and the positioning performance probably gets worse.
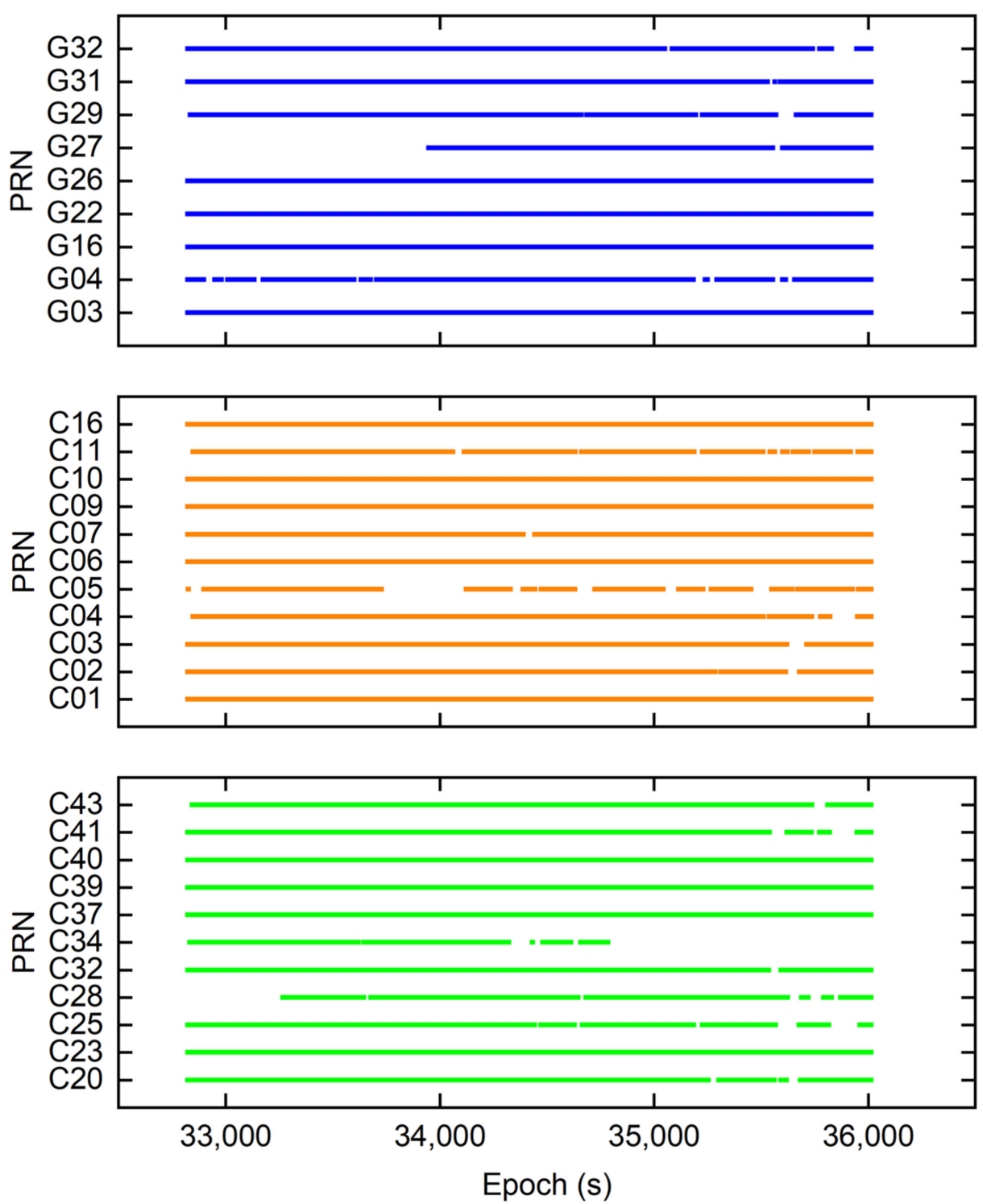

Figure 6. The tracked GPS/BDS satellites during the field test $\left(15^{\circ}\right.$ cut-off elevation angle).

Furthermore, we calculated the number of visible satellites for the single and combined systems and their PDOP values at every epoch, as displayed in Figure 7. It can be seen that, compared with the only GPS system, the number of available satellites significantly increased after combining with the BDS system, and the PDOP values decreased accordingly. In the meantime, we also found that the sudden decrease of the satellite numbers in several epochs immediately caused sharp increases of the PDOP values. This implies that the satellite number and the PDOP value were negatively correlated.

The subsequent statistical results showed that the average numbers of the GPS, BDS, and GPS/BDS satellites were 7.5, 17.1, and 23.8, and the corresponding average PDOP values were 2.21, 1.71, and 1.36, respectively. Compared with a single GPS, the average PDOP values of the BDS and the GPS+BDS combined system increased by $22.6 \%$ and $38.5 \%$. That is the reason why we selected the combined GPS/BDS system for data processing. 

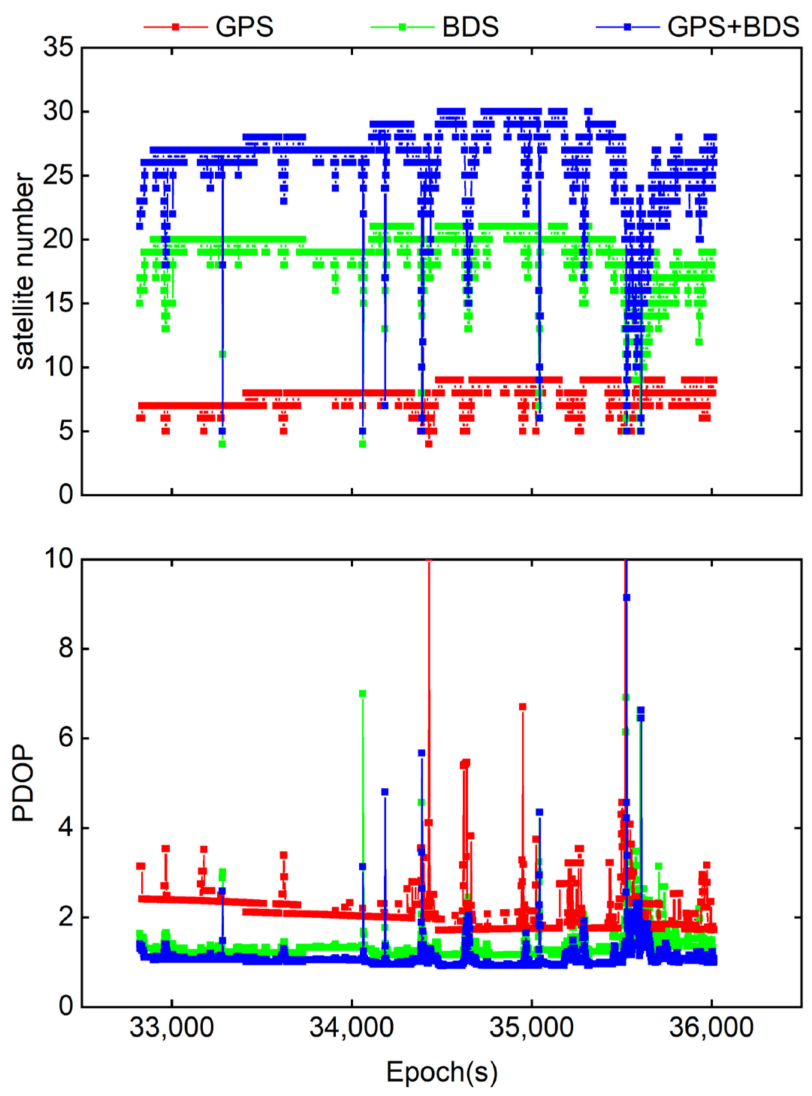

Figure 7. The number of visible satellites and the corresponding PDOP values, with a cut-off elevation angle of $15^{\circ}$.

\subsection{Evaluation of Position Variation}

In order to analyze and assess the performance of the TDCP/INS method in the stability of the position datum, the position results from TC RTK/INS were used as the reference. Meanwhile, the position variations between adjacent epochs were also obtained in the SPP, KFSPP, and TDCP methods for comparison. The position variations along the timeline in the SPP, KFSPP, TDCP, TDCP/INS, and the referenced TC RTK/INS methods are displayed in Figure 8.

From Figure 8, we can see that the position variations obtained from the TC RTK/INS method ranged within $15 \mathrm{~m}$ in the east/north direction and $1 \mathrm{~m}$ in the upward direction, and these results were regarded as the reference values. The results obtained from the TDCP/INS method were much similar compared to the reference, which means that the TDCP/INS method also accurately described the actual motion state of the moving devices. Comparatively, although the TDCP-only method achieved similar position variations along with the time, there were obviously many more outliers during the whole experiment, especially in the upward direction. This means that additional INS can remarkably improve the reliability of the solution in severe environments.

On the contrary, the results from the SPP method showed lots of outliers larger than $20 \mathrm{~m}$, which means a poor accuracy was achieved. The results from the KFSPP method were slightly better, but there were also a few outliers at about $10 \mathrm{~min}$ before the end of the field test. These could be easily distinguished from the referenced results by the TC RTK/INS method.

Furthermore, the position variations obtained from the SPP, KFSPP, TDCP, and TDCP/INS methods were differenced with the reference values, and the biases of their position variations during the field test were acquired and are shown in Figure 9. The bias results in the figure directly reflected the accuracies of the position variations obtained from the SPP, KFSPP, TDCP, and TDCP/INS methods. 

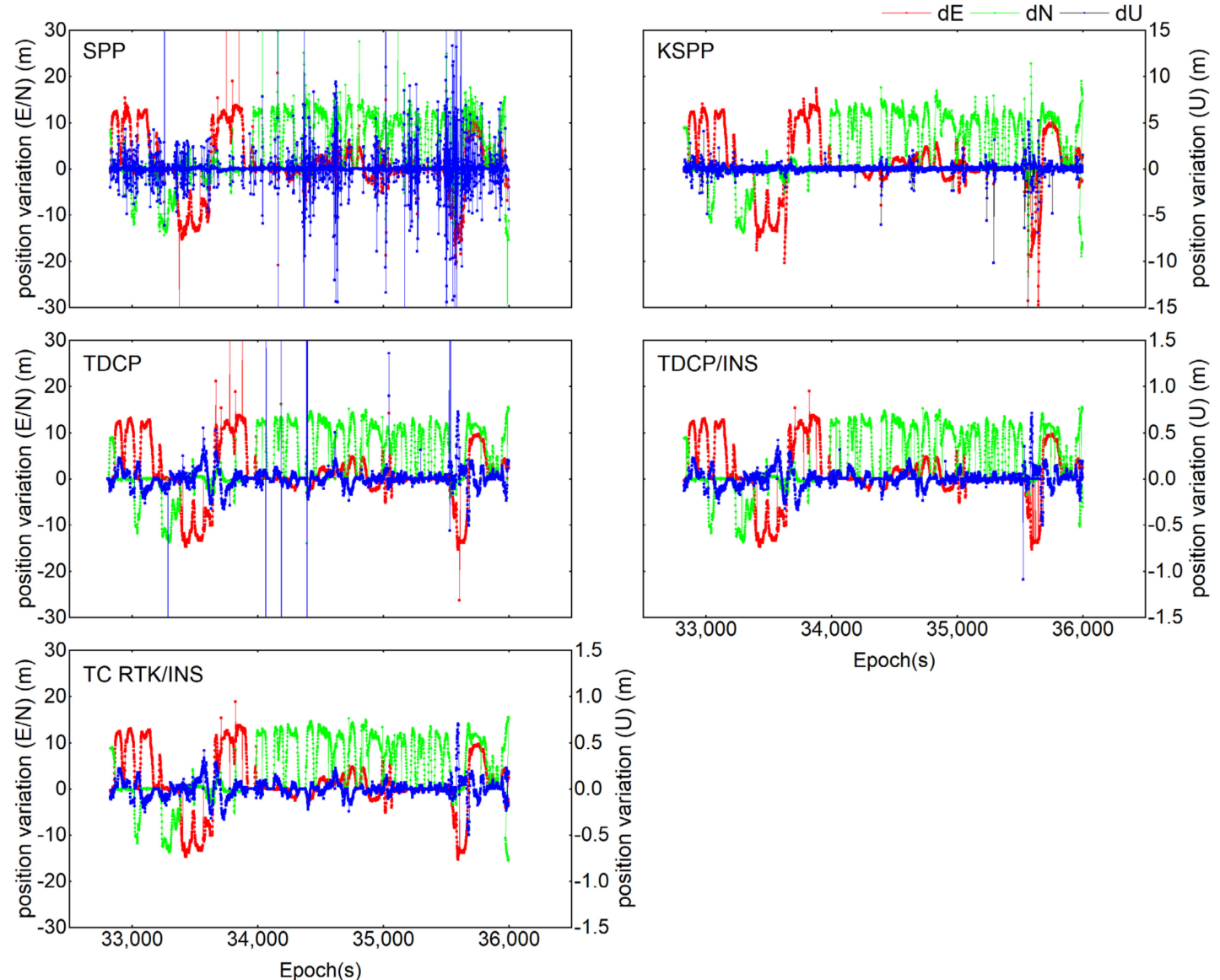

Figure 8. The position variations in the east/north/upward directions between adjacent epochs obtained from different methods during the field test (upward-left: SPP, upward-right: KFSPP, middle-left: TDCP, middle-right: TDCP/INS, and down-left: TC RTK/INS).
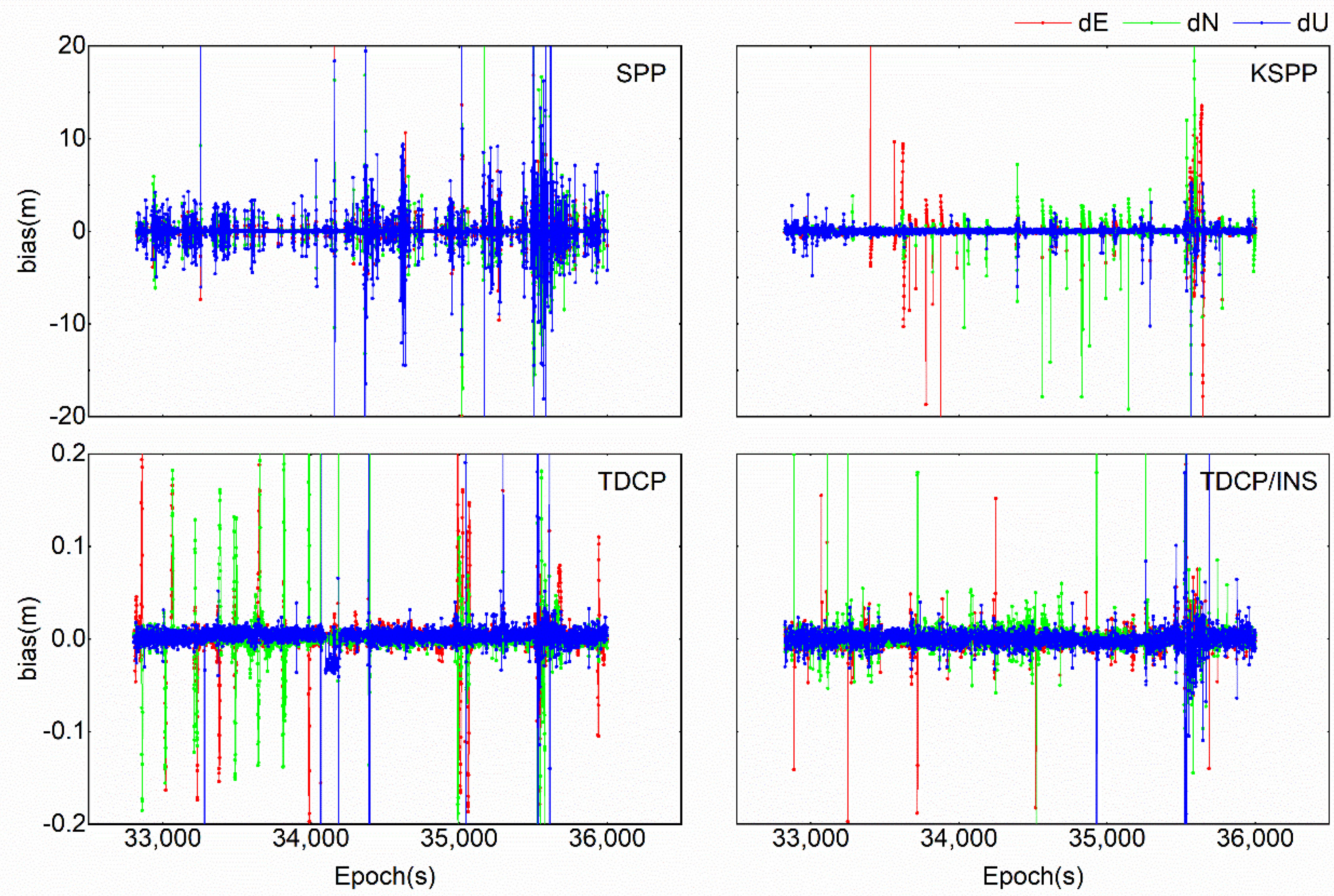

Figure 9. The biases of the position variations in the east/north/upward directions during the field test where the results from TC RTK/INS were regarded as the benchmarks. 
Generally speaking, the SPP method achieved the worst accuracy, and the KFSPP method had a moderate improvement. Comparatively, the TDCP and TDCP/INS methods achieved more accurate results. As expected, almost 100 times better results were obtained from the TDCP/INS method than those from the SPP method. Due to the lack of INS, there were many more outliers in the TDCP results than the TDCP/INS results, and its robustness decreased. The biases from the TDCP/INS method were almost limited within only $0.05 \mathrm{~m}$, and this performance was comparable to that in the popular RTK positioning.

For a quantitative analysis, the standard deviation (STD) and root mean square (RMS) values of the position variations obtained from the four methods were calculated and are listed in Table 4. From the table, we can find that, compared with the results from the TC RTK/INS method, the biases of the position variations in both the SPP and the KFSPP were at the meter level. Surprisingly, the biases of the position variations in the TDCP method were still at the meter level, with only a slight decrease compared to the KFSPP method. This should be attributed to the poor quality of the GNSS signals in severe environments, which lead to a lot of outliers. With the help of the INS data, the biases in the TDCP/INS method were dramatically reduced to the centimeter level. The STD values and the RMS values were much similar for all three methods, which implies the results from different methods are consistent regardless of their noise differences.

Table 4. STD and RMS values of the position variations obtained from different methods.

\begin{tabular}{ccccccc}
\hline \multirow{2}{*}{ Method } & \multicolumn{3}{c}{ STD $(\mathbf{m})$} & \multicolumn{3}{c}{ RMS (m) } \\
\cline { 2 - 7 } & $\mathbf{E}$ & $\mathbf{N}$ & $\mathbf{U}$ & $\mathbf{E}$ & $\mathbf{N}$ & $\mathbf{U}$ \\
\hline SPP & 1.148 & 1.634 & 2.499 & 1.148 & 1.635 & 2.499 \\
KFSPP & 0.779 & 1.523 & 1.754 & 0.780 & 1.523 & 1.754 \\
TDCP & 0.693 & 1.207 & 1.407 & 0.694 & 1.207 & 1.408 \\
TDCP /NS & 0.019 & 0.028 & 0.031 & 0.019 & 0.029 & 0.031 \\
\hline
\end{tabular}

Specifically, compared with the worst results from the SPP method, the accuracies of the position variations obtained from the KFSPP and TDCP methods had considerable improvements of about $32.1 \%, 6.9 \%$, and $29.8 \%$ and $39.5 \%, 26.2 \%$, and $43.7 \%$ in the east/north/upward directions, respectively, and that, from the TDCP/INS method, improved by nearly 100 times. The STD and RMS values of the TDCP/INS method reached the centimeter level, and the accuracies were $1.9 \mathrm{~cm}, 2.9 \mathrm{~cm}$, and $3.1 \mathrm{~cm}$ in the east/north/upward directions. As a result, the TDCP/INS method obtained the most accurate position variations and, thus, the most stable moving reference positions.

\subsection{Analysis of the Trajectory Consistency}

After the quantitative analysis of the accuracy of the position variations from the SPP, KFSPP, and TDCP/INS methods, we further made a qualitative analysis of the trajectory consistencies from these methods. The ground trajectory of the vehicle obtained from the TC RTK/INS method was used as the benchmark trajectory, and the position results obtained from the SPP, KFSPP, TDCP, and TDCP/INS methods were compared with the benchmarks to analyze their consistencies.

For the above purpose, three typical urban driving scenarios, which included going along a large, curved road, turning at a crossroad, and passing under an urban viaduct, were selected for the discussion. The comparative vehicle trajectories obtained from the different methods with respect to the benchmarks from the TC RTK/INS method in the above three scenarios are demonstrated from Figures 10-12, where the red pins, the blue pins, the green pins, the orange pins, and the yellow pins represent the position results from the TC RTK/INS method, the SPP method, the KFSPP method, the TDCP method, and the TDCP/INS method, respectively. 


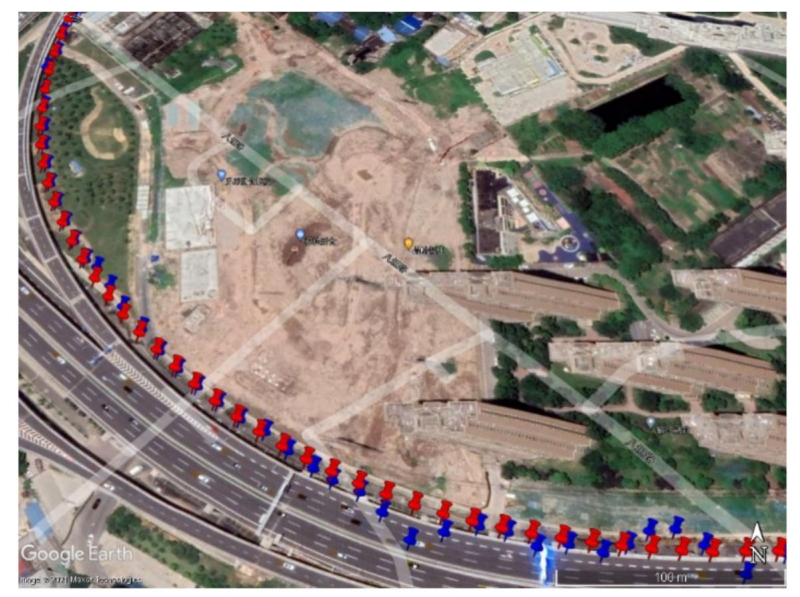

(a)

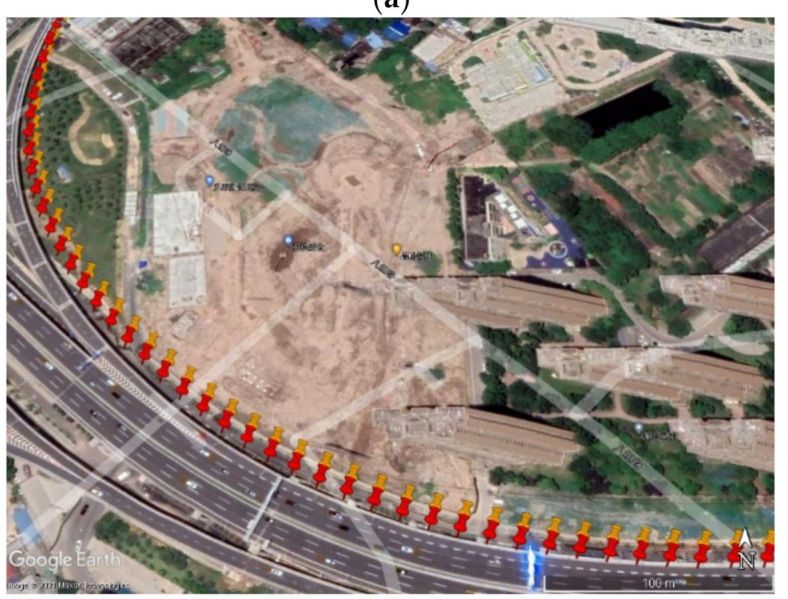

(c)

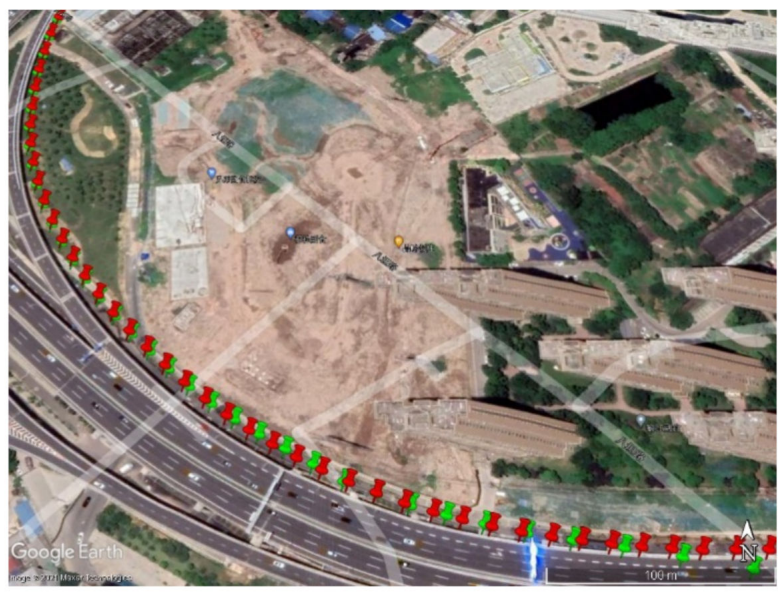

(b)

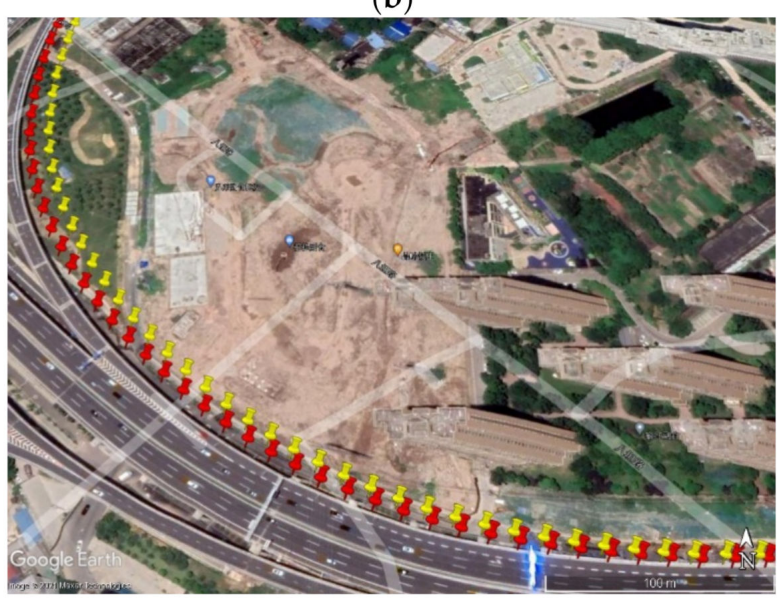

(d)

Figure 10. Comparison of the vehicle trajectories from different methods with the benchmarks by TC RTK/INS (red pins) when the vehicle went along a large, curved road: (a) SPP results (blue pins), (b) KFSPP results (green pins), (c) TDCP results (orange pins), and (d) TDCP/INS results (yellow pins). Longitude and latitude range from $114^{\circ} 16^{\prime} 44^{\prime \prime} \mathrm{E}, 30^{\circ} 29^{\prime} 32^{\prime \prime} \mathrm{N}$ to $114^{\circ} 17^{\prime} 00^{\prime \prime} \mathrm{E}, 30^{\circ} 29^{\prime} 43^{\prime \prime} \mathrm{N}$.

From Figures 10 and 11, we can see that the ground trajectory with the red pins obtained from the TC RTK/INS method was very smooth when the vehicle went along the curved road and turned at the crossroad. Additionally, the density of the red pins also reflected the driving velocity of the vehicle, since the pins in the figures are marked in equal time intervals of $1 \mathrm{~s}$. Therefore, it clearly reflects in Figure 10 that the vehicle went along the curved road at an almost-constant speed, while we found obvious deceleration and acceleration of the vehicle before and after it turned at the cross in Figure 11. Note that traffic keeps on the right in China. From this perspective, the position results from the TC RTK/INS method can be reliably regarded as the "true" trajectory.

We first investigated the blue pins in Figure 10a, which denoted the results from the SPP method, and found obvious jumps along the true trajectory when the vehicle drove from the east-south direction into the curved section. In Figure 11a, the blue pins changed from the left side of the true trajectory to its right side when the vehicle turned at the cross. All these results imply that inaccurate results were obtained from the SPP method.

The green pins, which denoted the results from the KFSPP method, had a moderate improvement compared with the blue pins. Few jumps could be found for the green pins in Figure 10b, but the changes from the left side of the true trajectory to its right side were still observed when the vehicle turned at the cross in Figure 11b, just like what the blue pins showed. This indicated that the accuracy of the KFSPP method was still unsatisfactory. 


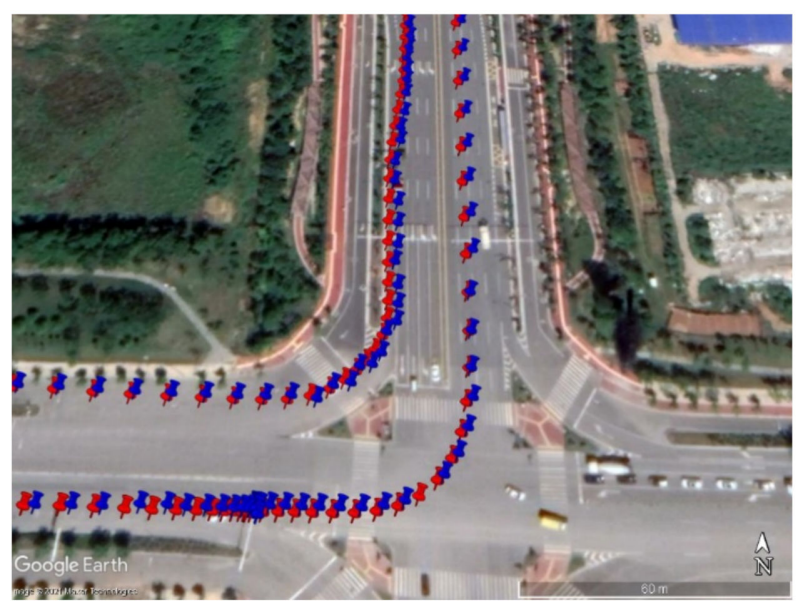

(a)

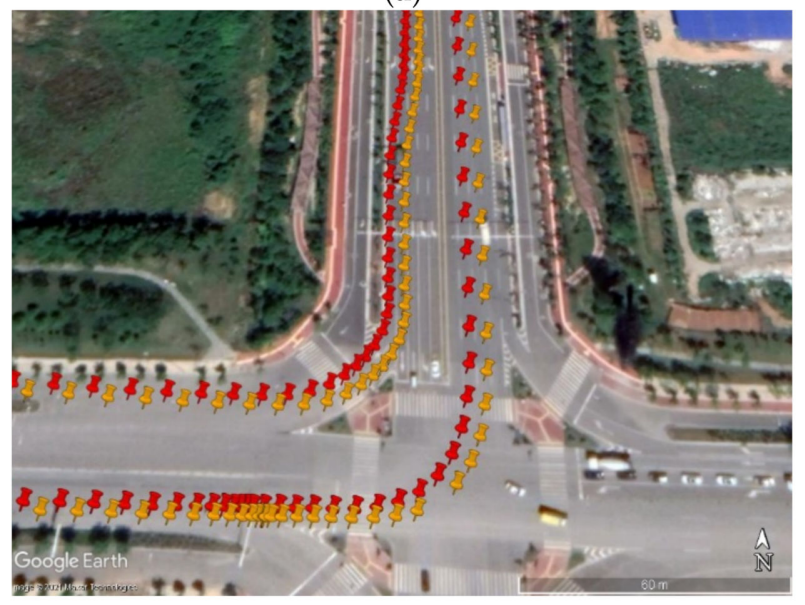

(c)

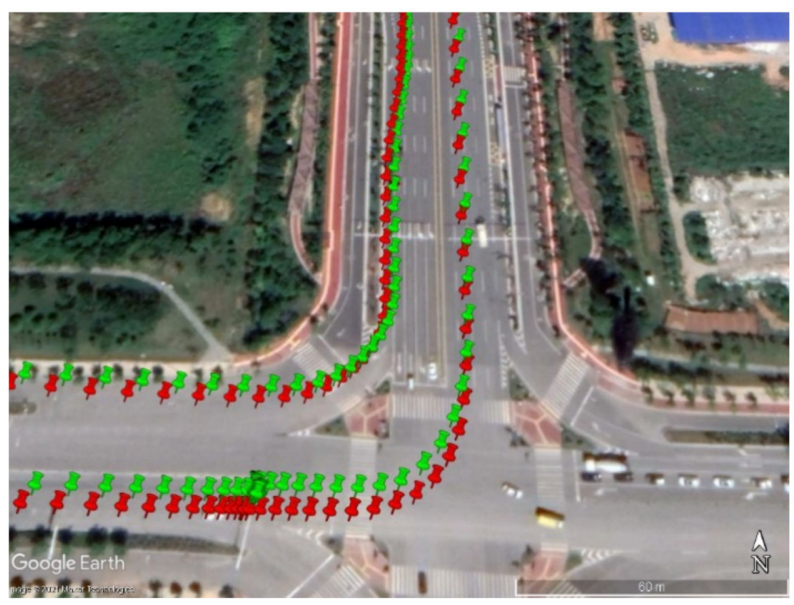

(b)

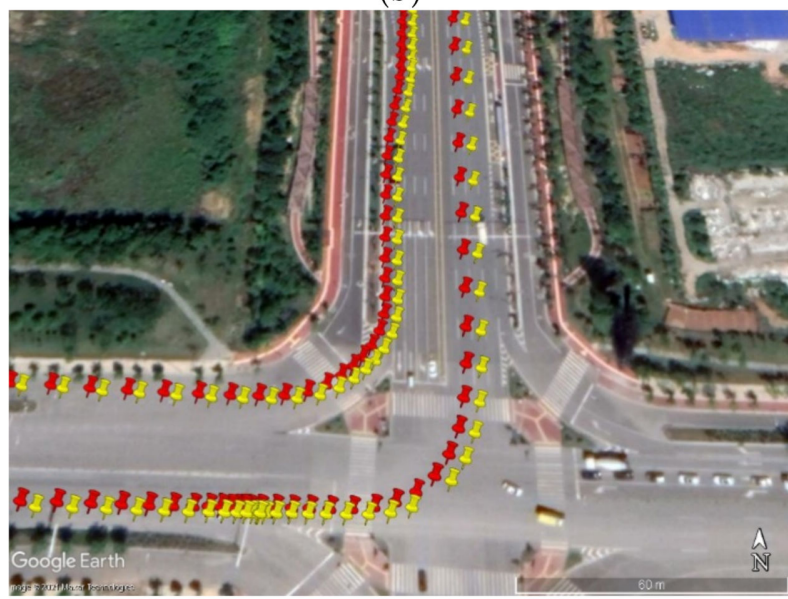

(d)

Figure 11. Comparison of the vehicle trajectories from different methods with the benchmarks by TC RTK/INS (red pins) when turning at a crossroad: (a) SPP results (blue pins), (b) KFSPP results (green pins), (c) TDCP results (orange pins), and (d) TDCP/INS results (yellow pins). Longitude and latitude range $114^{\circ} 17^{\prime} 17^{\prime \prime} \mathrm{E}, 30^{\circ} 22^{\prime} 42^{\prime \prime} \mathrm{N}$ to $114^{\circ} 17^{\prime} 25^{\prime \prime} \mathrm{E}, 30^{\circ} 22^{\prime} 48^{\prime \prime} \mathrm{N}$.

In contrast, the orange and yellow pins, which denoted the results from the TDCP and TDCP/INS methods, were very consistent with the red pins, despite a fixed offset between them in Figures 10c,d and 11c,d. We also understand that the offset was caused by the SPP result in the very beginning epoch and had no effect on the trajectory consistency. Additionally, the orange and yellow pins had almost the same densities as that for the red pins, which demonstrated that the TDCP and TDCP/INS methods could achieve almost the same levels of performance as the benchmarks from the TC RTK/INS method in open environments.

The position results when the vehicle passed under a viaduct demonstrated the performances of different methods when the signals were seriously interrupted, as can be seen in Figure 12. Poor positioning results were still witnessed for the SPP method and the KFSPP method, since the trajectories formed by the blue pins in Figure 12a and the green pins in Figure 12b swayed left and right along the true trajectory formed by the red pins. In contrast to the results from the former two scenarios, in the trajectory formed by the orange pins in Figure 12c, there were also jumps around the true trajectory, just like the SPP and KFSPP methods. Additionally, there were result interruptions when the vehicle just passed under the viaduct for all four methods. This was caused by the loss of satellite signals and could be confirmed by the number of visible satellites in Figure 7. The limited satellite number made positioning impossible in that epoch. Fortunately, the position results of the 
TDCP/INS method were right immediately after the vehicle passed the viaduct, and its trajectory was almost in parallel with the true trajectory.

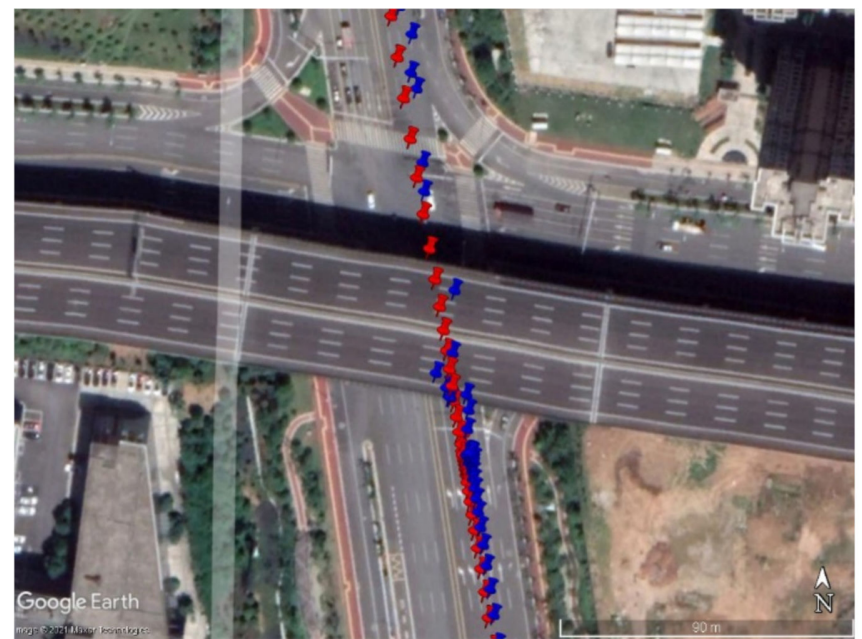

(a)

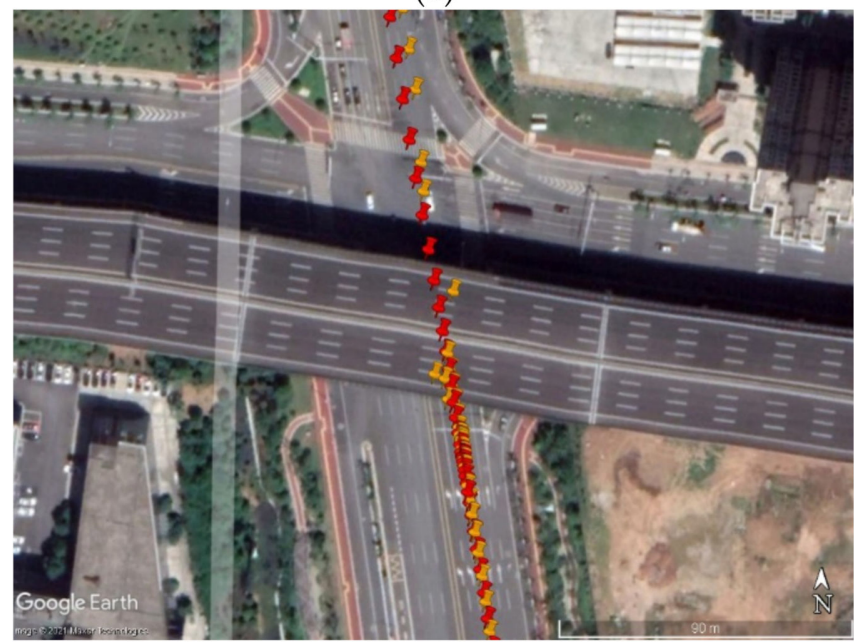

(c)

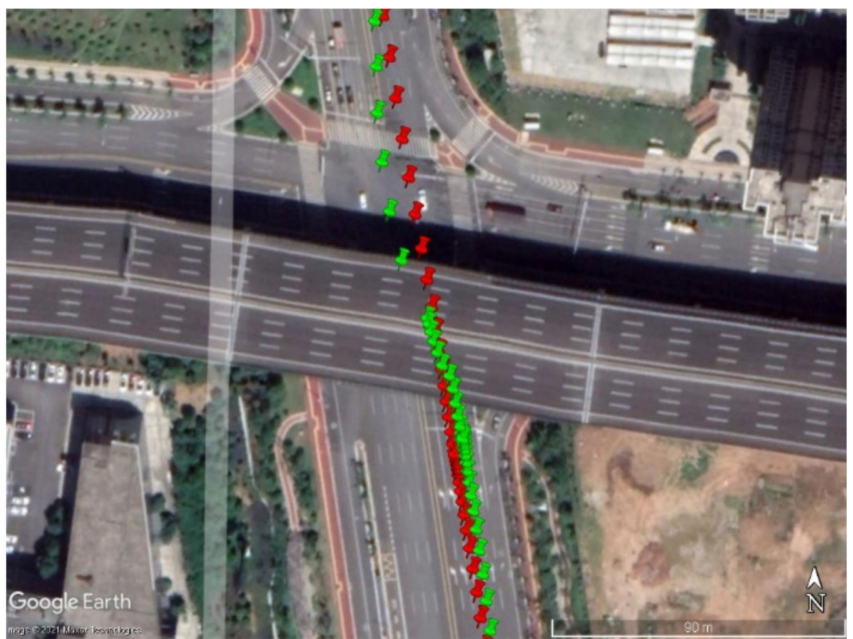

(b)

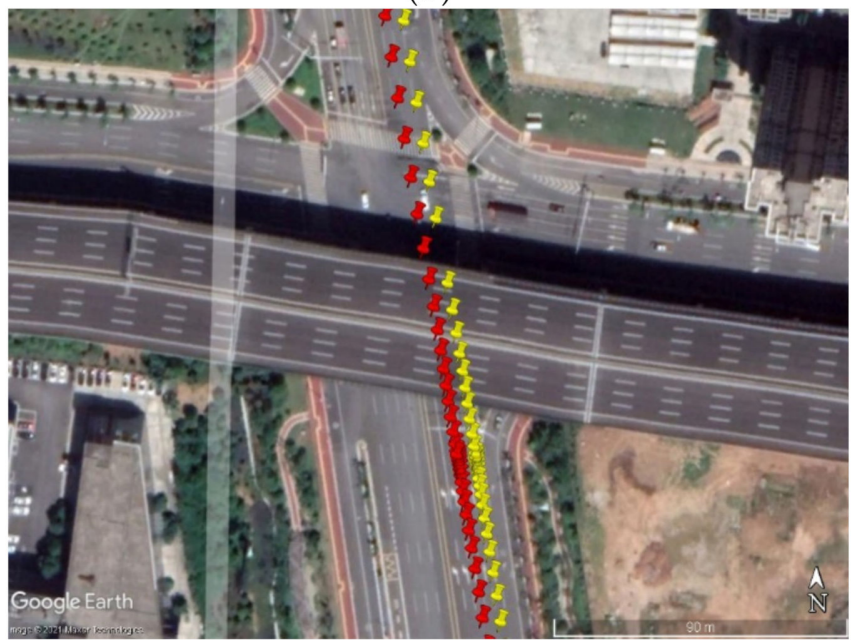

(d)

Figure 12. Comparison of the vehicle trajectories from different methods with the benchmarks by TC RTK/INS (red pins) when the vehicle passed under an urban viaduct: (a) SPP results (blue pins), (b) KFSPP results (green pins), (c) TDCP results (orange pins), and (d) TDCP/INS results (yellow pins). Longitude and latitude range from $114^{\circ} 17^{\prime} 14^{\prime \prime} \mathrm{E}, 30^{\circ} 24^{\prime} 18^{\prime \prime} \mathrm{N}$ to $114^{\circ} 17^{\prime} 23^{\prime \prime} \mathrm{E}, 30^{\circ} 24^{\prime} 24^{\prime \prime} \mathrm{N}$.

Since the position results from the TDCP/INS method in a single moving device were consistent with the ground true trajectory, the kinematic positioning results of the entire moving platform system were also consistent with the ground true trajectory. From this perspective, the TDCP/INS method realized the stable position datum and obtained a consistent moving trajectory with the true one without dependency on additional static reference stations.

\section{Discussion}

In the "static and dynamic" mode for a moving platform system, the coordinate of the reference station is fixed as the position datum of the entire platform system. Therefore, no matter how the platform system moves, once the relative positions are determined by the kinematic relative positioning, the position of every device in the platform system can be referred to the static position datum, and the positioning results of all devices must be consistent with their true motion trajectories. 
However, in the "kinematic-to-kinematic" mode, the inaccurate coordinates of the moving reference device obtained through the noisy SPP or KFSPP methods will cause a stochastic swaying of the entire platform system, along with the time, despite that the relative positions of each device in the system can be accurately described. The key issue lies in the inconsistency between the selected position datum of the moving platform system and its true position. Since accurate relative position can be easily obtained, this inconsistency will eventually lead to inconsistency between the positioning results of the entire moving platform system and the actual motion trajectory. As a consequence, the safe distance and coordinated operation between each device in the moving platform system cannot be reliably guaranteed.

The alternative solution proposed in this paper chose to determine the position variations between adjacent epochs rather than obtain the direct position information. The TDCP observations were applied to obtain accurate position variations, and then, the position of the moving device could be precisely updated. The INS data was further integrated with the GNSS-derived position to enhance the reliability of the updated position. This indirect method demonstrated a good performance in the field test.

Once the position datum of the moving platform system became stable, without dependency on additional static reference stations, a novel operational pattern for a platform system with multiple moving devices was expected. In this pattern, any moving devices in the platform system could be regarded as the position datum for itself and acquire the relative positions of the nearby devices. It was clearly shown that this pattern was decentralized, and the breakdown of any devices in the system had no effect on the normal operation of other devices and the entire platform system. This is very different from the current pattern, in which the system operation relies a lot on the static reference station near the platform. From this perspective, the expected pattern was more robust in the system level.

\section{Conclusions}

In the conventional relative positioning in a platform system with multiple moving devices, the positioning results obtained by the SPP or KFSPP methods were inconsistent with the actual motion trajectory due to the instability of the position datum. To solve this problem, we proposed the TDCP/INS method to transfer and update the position datum of the moving platform via the position variations from the TDCP observations and the INS data. The positioning results of the moving platform were consistent with the actual motion trajectory.

A field test using a driving vehicle was carried out, and the performance of the proposed TDCP/INS method was analyzed and compared with the SPP method, KFSPP method, TDCP method, and the referenced TC RTK/INS method. The experiment results showed that there were large biases of the position variations for the SPP and KFSPP methods. The accuracies of the calculated position variations for the SPP method were $1.148 \mathrm{~m}, 1.635 \mathrm{~m}$, and $2.499 \mathrm{~m}$ in the east/north/upward directions, which, for the KFSPP and TDCP methods, had improvements of $32.1 \%, 6.9 \%$, and $29.8 \%$ and $39.5 \%, 26.2 \%$, and $43.7 \%$, and which, for the TDCP/INS method, improved by nearly 100 times and reached the centimeter level.

A further qualitative analysis demonstrated the perfect consistency between the position results from the TDCP/INS method and the reference positions in three typical urban driving scenarios, including going along a large, curved road, turning at a crossroad, and passing under an urban viaduct. The motion states of the vehicle, such as the acceleration and deceleration, were also reflected with the TDCP/INS method. On the contrary, there were frequent jumps and sways along the driving direction, and obvious outliers of the positioning results were detected with the SPP, KFSPP, and TDCP methods. The comparative analysis indicated an outstanding positioning performance from the proposed TDCP/INS method, which could achieve a stable position datum in the platform system with multiple moving devices. 
In a future work, a platform system with UAVs will be established, and a corresponding field test will be carried out. Additionally, quality control algorithms for the INS data, especially the blunder detection and noise elimination, are planned to be employed to further improve the stability and reliability of the positioning results.

Author Contributions: Conceptualization, W.T. and Y.L.; methodology, C.D.; software, W.T.; validation, Y.L., Y.W. and X.Z.; formal analysis, C.D.; investigation, Y.L.; resources, X.Z.; data curation, Y.L.; writing —original draft preparation, W.T.; writing—review and editing, C.D. and Y.L.; visualization, Y.L. and K.Q.; supervision, W.T.; project administration, W.T.; and funding acquisition, W.T. and C.D. All authors have read and agreed to the published version of the manuscript.

Funding: This research was funded by China's major projects of the second-generation satellite navigation system (grant no. GFZX030302030205-1), Joint Fund of the Ministry of Education of China for Equipment Pre-research (grant no. 6141A02011907), the National Natural Science Foundation of China (grant nos. 41804028 and 41874037), and the Wuhan Science and Technology Project (grant no. 2018010401011271).

Institutional Review Board Statement: Not applicable.

Informed Consent Statement: Not applicable.

Data Availability Statement: The data in this paper are available from the corresponding author for academic purposes upon reasonable request.

Acknowledgments: We thank the three anonymous reviewers for their constructive comments that helped to improve the quality of this paper.

Conflicts of Interest: The authors declare no conflict of interest.

\section{References}

1. Kouba, J.; Héroux, P. Precise Point Positioning Using IGS Orbit and Clock Products. GPS Solut. 2001, 5, 12-28. [CrossRef]

2. Bisnath, S.; Gao, Y. Current state of precise point positioning and future prospects and limitations. In Observing Our Changing Earth; Sideris, M.G., Ed.; Springer: Berlin/Heidelberg, Germany, 2009; pp. 615-623. [CrossRef]

3. Elsobeiey, M.; Alharbi, S.M. Performance of real-time Precise Point Positioning using IGS real-time service. GPS Solut. 2016, 20 565-571. [CrossRef]

4. Han, S.; Rizos, C. GPS Network Design and Error Mitigation For Real-Time Continuous Array Monitoring System. In Proceedings of the 9th International Technical Meeting of the Satellite Division of US Inst Navigation, Kansas City, MO, USA, 17-20 September 1996; pp. 1827-1836. [CrossRef]

5. Luo, N. Centimetre-Level Relative Positioning of Multiple Moving Platforms Using Ambiguity Constraints. In Proceedings of the 13th International Technical Meeting of the Satellite Division of The Institute of Navigation (ION GPS 2000), Salt Lake City, UT, USA, 19-22 September 2000; pp. 113-122. [CrossRef]

6. Takasu, T.; Yasuda, A. Development of the Low-Cost RTK-GPS Receiver with an Open Source Program Package RTKLIB. In Proceedings of the International Symposium on GPS/GNSS, Jeju, Korea, 4-6 November 2009. [CrossRef]

7. Li, T.; Zhang, H.; Niu, X.; Gao, Z. Tightly-Coupled Integration of Multi-GNSS Single-Frequency RTK and MEMS-IMU for Enhanced Positioning Performance. Sensors 2017, 17, 2462. [CrossRef] [PubMed]

8. Berber, M.; Munjy, R.; Lopez, J. Kinematic GNSS positioning results compared against Agisoft Metashape and Pix4dmapper results produced in the San Joaquin experimental range in Fresno County, California. J. Géod. Sci. 2021, 11, 48-57. [CrossRef]

9. Gurturk, M.; Soycan, M. Accuracy assessment of kinematic PPP versus PPK for GNSS flights data processing. Surv. Rev. 2021, 1-9. [CrossRef]

10. Wu, Q.; Sun, M.; Zhou, C.; Zhang, P. Precise Point Positioning Using Dual-Frequency GNSS Observations on Smartphone. Sensors 2019, 19, 2189. [CrossRef]

11. Gianey, H.K.; Ali, M.; Vijayakumar, V.; Sharma, A.; Kumar, R. Low Cost and Centimeter-Level Global Positioning System Accuracy Using Real-Time Kinematic Library and Real-Time Kinematic GPSA. Recent Adv. Comput. Sci. Commun. 2021, 14, 360-367. [CrossRef]

12. Luo, N. Precise Relative Positioning of Multiple Moving Platforms Using Gps Carrier Phase Observables. Ph.D. Thesis, University of Calgary, Calgary, AB, Canada, 2001.

13. Dong, Y.; Zhang, L.; Wang, D.; Li, Q.; Wu, J.; Wu, M. Low-latency, high-rate, high-precision relative positioning with moving base in real time. GPS Solut. 2020, 24, 56. [CrossRef]

14. Feng, Y.; Wang, J. GPS RTK Performance Characteristics and Analysis. J. Glob. Position. Syst. 2008, 7, 1-8. [CrossRef]

15. Deng, C.; Tang, W.; Liu, J.; Shi, C. Reliable single-epoch ambiguity resolution for short baselines using combined GPS/BeiDou system. GPS Solut. 2014, 18, 375-386. [CrossRef] 
16. Innac, A.; Angrisano, A.; Dardanelli, G.; Della Corte, V.; Martellato, E.; Rotundi, A.; Ferraioli, G.; Palumbo, P.; Gaglione, S. A Kalman filter single point positioning for maritime applications using a smartphone. Geogr. Tech. 2020, 16, 15-29. [CrossRef]

17. RTCM Special Committee. RTCM Standard 10403.3 Differential GNSS (Global Navigation Satellite Systems) Services-Version 3; RTCM: Arlington, TX, USA, 2016.

18. Montenbruck, O.; Steigenberger, P.; Prange, L.; Deng, Z.; Zhao, Q.; Perosanz, F.; Romero, I.; Noll, C.; Stürze, A.; Weber, G.; et al. The Multi-GNSS Experiment (MGEX) of the International GNSS Service (IGS)-Achievements, prospects and challenges. Adv. Space Res. 2017, 59, 1671-1697. [CrossRef]

19. Salazar, D.; Hernandez-Pajares, M.; Juan-Zornoza, J.M.; Sanz-Subirana, J.; Aragon-Angel, A. EVA: GPS-based extended velocity and acceleration determination. J. Geod. 2011, 85, 329-340. [CrossRef]

20. Soon, B.K.H.; Scheding, S.; Lee, H.-K.; Lee, H.-K.; Durrant-Whyte, H. An approach to aid INS using time-differenced GPS carrier phase (TDCP) measurements. GPS Solut. 2008, 12, 261-271. [CrossRef]

21. Wendel, J.; Trommer, G.F. Tightly coupled GPS/INS integration for missile applications. Aerosp. Sci. Technol. 2004, 8, 627-634. [CrossRef]

22. Freda, P.; Angrisano, A.; Gaglione, S.; Troisi, S. Time-differenced carrier phases technique for precise GNSS velocity estimation. GPS Solut. 2015, 19, 335-341. [CrossRef]

23. Van Graas, F.; Soloviev, A. Precise Velocity Estimation Using a Stand-Alone GPS Receiver. Navigation 2004, 51, 283-292. [CrossRef]

24. Groves, P.D. Principles of GNSS, Inertial, and Multisensor Integrated Navigation Systems, 2nd ed.; Artech House: London, UK, 2013 [CrossRef]

25. Lee, J.Y.; Kim, H.S.; Choi, K.H.; Lim, J.; Chun, S.; Lee, H.K. Adaptive GPS/INS integration for relative navigation. GPS Solut. 2015, 20, 63-75. [CrossRef]

26. Faulkner, N.; Cooper, S.; Jeary, P. Integrated MEMS/GPS Navigation Systems. In Proceedings of the 2002 IEEE Position Location and Navigation Symposium (IEEE Cat. No.02CH37284), Palm Springs, CA, USA, 15-18 April 2002; pp. 306-313. [CrossRef]

27. Niu, X.; Nassar, S.; El-Sheimy, N. An Accurate Land-Vehicle MEMS IMU/GPS Navigation System Using 3D Auxiliary Velocity Updates. Navigation 2007, 54, 177-188. [CrossRef]

28. El-Sheimy, N.; Youssef, A. Inertial sensors technologies for navigation applications: State of the art and future trends. Satell. Navig. 2020, 1, 2. [CrossRef]

29. Li, T.; Zhang, H.; Gao, Z.; Chen, Q.; Niu, X. High-Accuracy Positioning in Urban Environments Using Single-Frequency Multi-GNSS RTK/MEMS-IMU Integration. Remote Sens. 2018, 10, 205. [CrossRef]

30. Chiang, K.-W.; Duong, T.T.; Liao, J.-K. The Performance Analysis of a Real-Time Integrated INS/GPS Vehicle Navigation System with Abnormal GPS Measurement Elimination. Sensors 2013, 13, 10599-10622. [CrossRef]

31. Klobuchar, J.A. Ionospheric Time-Delay Algorithm for Single-Frequency GPS Users. IEEE Trans. Aerosp. Electron. Syst. 1987, AES-23, 325-331. [CrossRef]

32. Saastamoinen, J. Contributions to the theory of atmospheric refraction. Bull. Géod. 1972, 105, 279-298. [CrossRef]

33. Kim, J.; Park, M.; Bae, Y.; Kim, O.-J.; Kim, D.; Kim, B.; Kee, C. A Low-Cost, High-Precision Vehicle Navigation System for Deep Urban Multipath Environment Using TDCP Measurements. Sensors 2020, 20, 3254. [CrossRef]

34. Kim, Y.; Song, J.; Kee, C.; Park, B. GPS Cycle Slip Detection Considering Satellite Geometry Based on TDCP/INS Integrated Navigation. Sensors 2015, 15, 25336-25365. [CrossRef]

35. Blewitt, G. An Automatic Editing Algorithm for GPS data. Geophys. Res. Lett. 1990, 17, 199-202. [CrossRef]

36. Niu, X.; Zhang, Q.; Gong, L.; Liu, C.; Zhang, H.; Shi, C.; Wang, J.; Coleman, M. Development and evaluation of GNSS/INS data processing software for position and orientation systems. Surv. Rev. 2015, 47, 87-98. [CrossRef] 